Delft University of Technology

\title{
Micro-Doppler Period Estimation Based on Concentration Statistics of Ambiguity Function
}

Zhang, Wenpeng; Fu, Yaowen; Yin, Jiapeng

DOI

10.1109/TAES.2019.2921192

Publication date

2020

Document Version

Accepted author manuscript

Published in

IEEE Transactions on Aerospace and Electronic Systems

\section{Citation (APA)}

Zhang, W., Fu, Y., \& Yin, J. (2020). Micro-Doppler Period Estimation Based on Concentration Statistics of Ambiguity Function. IEEE Transactions on Aerospace and Electronic Systems, 56(3), 1722-1741.

[8759924]. https://doi.org/10.1109/TAES.2019.2921192

\section{Important note}

To cite this publication, please use the final published version (if applicable).

Please check the document version above.

\section{Copyright}

Other than for strictly personal use, it is not permitted to download, forward or distribute the text or part of it, without the consent of the author(s) and/or copyright holder(s), unless the work is under an open content license such as Creative Commons.

\section{Takedown policy}

Please contact us and provide details if you believe this document breaches copyrights.

We will remove access to the work immediately and investigate your claim. 


\title{
Micro-Doppler Period Estimation based on Concentration Statistics of Ambiguity Function
}

\author{
Wenpeng Zhang, Yaowen Fu, and Jiapeng Yin
}

\begin{abstract}
Radar micro-Doppler (m-D) signature, which reflects the micro-motion dynamic and structural characteristics of radar target with micro-motion, has been received increasing attention. Most of the existing m-D signature-extraction methods operate in the time domain or the time-frequency domain. Different from these methods, in this work, an m-D period estimation approach which operates in the ambiguity domain is proposed. Though the ambiguity function (AF) has been widely used in the field of radar signal processing, its application for $\mathrm{m}$-D signal is introduced for the first time. It is proved that the AF of m-D signal exhibits periodicity along the lag axis and has the best concentration when the lag equals to multiples of the $m-D$ period. Based on this, three AF concentration statistics are employed to capture the periodicity and to provide the $m$-D estimate. The most important property of the AF concentration statistics is that they are (or approximately) invariant to polynomial translations with terms not larger than second order even if the signal is Doppler ambiguous. Numeric simulation and real radar experiments are used to validate the effectiveness of the proposed technique.
\end{abstract}

Index Terms-Micro-Doppler, period estimation, ambiguity function, concentration statistics

\section{INTRODUCTION}

$\mathbf{R}$ ADAR target undergoing micro-motion dynamics imposes a periodic time-varying frequency modulation on radar signals. This is known as the micro-Doppler (m-D) effect [1]. The m-D features, which reflect the unique dynamic and structural characteristics of the target, serve as additional target features for target recognition and classification [1]-[3].

Numerous m-D signature-extraction methods have been proposed in the past decades. Time-frequency (TF) signal analysis, which provides a joint time-frequency representation (TFR) of a signal, is effective in describing the time-varying frequency modulation property of the m-D signals [1]. Thus, it is widely employed to analyze the m-D signatures.

For non-rigid radar targets, their main body signatures and $\mathrm{m}-\mathrm{D}$ signatures are superimposed. The m-D signal should be separated or extracted from the target's radar echo before further being processed. Generally, signal decomposition methods which decompose radar signals into components associated with different parts of radar target, e.g., chirplet decomposition [4], wavelet decomposition [5], empirical mode decomposition (EMD) [6], can be carried out to extract the m-D signatures.

W. Zhang and Y. Fu are with College of Electronic Science, National University of Defense Technology, 410073 Changsha, China. J. Yin is with College of Electronic Science, National University of Defense Technology, 410073 Changsha, China, and also with Delft University of Technology, 2628 Delft, the Netherlands (e-mail: zhangwenpeng@ hotmail.com; fuyaowen@nudt.edu.cn; j.yin@tudelft.nl). The corresponding author is Y. Fu.
The statistics of TFR is also effective in extracting the mD signatures. In [7], an L-statistics-based method for the mD effect removal was proposed and produced better focused images of the rigid body than other TF-based approaches.

After the extraction of the pure m-D signal, parameter estimation approaches can be applied to estimate the m-D period. Autocorrelation, which is a typical time-domain approach for period estimation, is useful for $\mathrm{m}-\mathrm{D}$ period estimation. This method was validated by the analysis of a helicopter target [5]. Based on the fact that the m-D signal presents periodicity on the TF plane, TF-domain approaches can be developed to estimate the m-D period [8]-[11]. Two approaches based on the one-dimensional Fourier transform [8] and the twodimensional Fourier transform [9] of the TFR were proposed to extract the m-D period (frequency), respectively. In [10], a similarity measure of the TFR, which is a two-dimensional correlation, was employed to estimate the micro-motion period. Taking advantage of the spectrum property of the m-D signal, the cyclic spectrum density (CSD) was proposed for the m$\mathrm{D}$ period estimation as well [11]. The aforementioned $\mathrm{m}$ D period estimation approaches were developed without the consideration of translation and may be invalid in the presence of translation.

In real scenarios, the micro-motion is often coupled with the translation (macro-motion) due to the relative bulk motion between the target and the radar platform [5], [9], [12], [13]. For aerial targets such as helicopter and ballistic warhead measured by the ground-based radar, the translation is identical to the target's motion which may be a constant (e.g., hovering helicopter) [5], a linear translation (the target is moving with constant velocity) [12] and a quadratic translation (the target is moving with constant acceleration) [9]. For ground targets such as rotating antenna measured by the airborne radar, the translation is often approximated by a quadratic translation [13].

In the presence of translation, the periodicity of the m-D signal is destroyed and the aforementioned $\mathrm{m}-\mathrm{D}$ period estimation methods in [5], [8]-[11] should be integrated with a translation compensation step. This means that the performances of these approaches largely depend on the translation compensation algorithms [14] and the computational complexity increases significantly because the translation compensation step usually involves a search for the unknown motion parameters. In a previous publication [15], the authors proved that in the presence of translation, the TFR of the m-D signal is circular shifted along the Doppler dimension, thus the periodicity of the TFR is transformed into the circular periodicity. Based 
on the circular periodicity property, the circular correlation coefficients of the TFR which can inherently remove the circular-Doppler-shift effect caused by the translation was proposed. In addition to the circular correlation coefficients, two different translation-compensation-free m-D period estimation methods, the time-frequency squared difference sequences of the scatterers' instantaneous frequencies [16] and the highorder difference sequence of the scatterer's instantaneous range [17], were proposed respectively.

Though these three methods have successfully achieved mD period estimation in the presence of translation, they still have limitations in processing $\mathrm{m}-\mathrm{D}$ signals with low signal-tonoise ratio (SNR). In addition, the computational efficiencies are low. This motivates the authors to explore a new m-D period estimation method.

This work focuses on m-D period estimation of radar targets with uniform and single-period micro-motion. Targets of interests include ballistic warhead, helicopter, wind turbines, rotating antenna, human, etc. Inspired by the high-order ambiguity function (HAF) [18] (which is good at processing polynomial-phase signals (PPSs)), this work proposes an m-D period estimation method based on the concentration statistics of the ambiguity function (AF). With theoretical derivation and careful analysis, the authors found that the AF has similar effect with the TF in terms of the translation. The AF is circular-Doppler-shifted in the presence of translation as well. Moreover, the concentration of the AF is periodic. Then, three concentration statistics including AF entropy, AF $l_{4}-l_{2}$ norm, and $\mathrm{AF} l_{1}$ norm which are invariant to the circularshift effect are proposed. Benefiting from the special property of the AF, the proposed method can provide the m-D period estimation without combining a translation compensation step. The contributions of this work include:

- The AF of m-D signal is discussed. Properties including the circular-Doppler-shift property of the AF in the presence of translation and the periodic concentration effect of the AF are uncoverd. An m-D period estimation method based on the concentration statistics of the AF is proposed, which enlarges the class of the m-D period estimation methods that are free of translation compensation.

- It has been widely believed that, in order to have a better performance for processing the multicomponent nonstationary m-D signal, TFRs without cross-term interference ${ }^{1}$ should be employed [1]. Our work presents a different way for the m-D period estimation and proves that the $\mathrm{AF}$ can do this as well although the signal suffers from cross-term interference.

- Comprehensive comparisons for existing m-D period estimation methods including estimation performance and computational complexity are provided. To the best of our knowledge, it is the first time in the field of m-D period estimation.

The rest of the paper is organized as follows. In Section II, the radar signal model and basic concepts of the m-D are

\footnotetext{
${ }^{1}$ The cross-terms of a signal are the coupling terms of its components, which are induced by the nonlinear functions/operators. The cross-terms may interfere some important features of a signal and are thought to be detrimental.
}

presented. Section III provides an overview of related work. The details of the proposed method are demonstrated in Section IV. Data verification consisting of a simulated coning target, a walking human, and a SAR experiment is given in Section V. Finally, Section VI draws some conclusions. A summary of the main notations is given in Table I.

\section{RADAR Signal MODEL}

Assuming that there are $L$ scatterers on the target, the radial distance of the $l$ th scatterer from radar can be expressed as

$$
r_{l}(t)=r_{T}(t)+r_{M, l}(t)
$$

where $r_{T}(t)$ is the range corresponding to the translation of the target, and $r_{M, l}(t)$ is the range corresponding to the individual micro-motion of the $l$ th scatterer, which meets

$$
r_{M, l}(t)=r_{M, l}\left(t+T_{M}\right)
$$

where $T_{M}$ is the micro-motion/micro-Doppler period of the target, and $f_{M}=1 / T_{M}$ is the micro-motion/micro-Doppler frequency.

A second-order-polynomial function is often enough to model the translation of a target [1]. The translation can be expressed as

$$
r_{T}(t)=R_{0}+\sum_{i=1}^{Q} a_{i} t^{i}
$$

where $R_{0}$ is the initial range. $Q$ may be 0,1 , and 2 corresponding to three scenarios respectively: micro-motion (MM for short), micro-motion plus linear translation (MMLT for short) and micro-motion plus quadratic translation (MMQT for short).

In general, the baseband signal of the returned radar signal can be expressed as

$$
s(t)=\sum_{l=1}^{L} \sigma_{l}(t) \exp \left(-\frac{j 4 \pi r_{l}(t)}{\lambda}\right)=s_{T}(t) s_{M}(t)
$$

where

$$
\begin{gathered}
s_{T}(t)=\exp \left(-\frac{j 4 \pi r_{T}(t)}{\lambda}\right) \\
s_{M}(t)=\sum_{l=1}^{L} \sigma_{l}(t) \exp \left(-\frac{j 4 \pi r_{M, l}(t)}{\lambda}\right) .
\end{gathered}
$$

$s_{T}(t)$ is the translation modulation component, $s_{M}(t)$ is the micro-motion modulation component, $\sigma_{l}(t)$ denotes the radar cross section (RCS) of the $l$ th scatterer, and $\lambda$ is the wavelength of the carrier wave. Specially, when $Q=0$, $s_{T}(t)=\exp \left(-\frac{j 4 \pi R_{0}}{\lambda}\right)$ is a constant and can be absorbed into $\sigma_{l}(t)$.

The RCS of a complex target is a function of aspect angle and frequency, and the aspect angle varies with the motion of the target. In most micro-motion research, periodic RCS is adopted, which simplifies the analysis of m-D signal [1],

$$
\sigma_{l}(t)=\sigma_{l}\left(t+T_{M}\right) .
$$

This assumption is violated when the aspect angle of the target fluctuates severely. Since the proposed method mainly 
TABLE I

NOTATIONS USED IN THIS WORK.

\begin{tabular}{cl||cl}
\hline \hline$t(n)$ & time variable (discrete time variable) & $\tau(m)$ & lag variable (discrete lag variable) \\
$f(k)$ & frequency variable (discrete frequency variable) & $r_{T}(t)$ & Radial distance corresponding to the translation of the target \\
$s(t)$ & the m-D signal to be analyzed & $s(n)$ & discrete version of the m-D signal to be analyzed \\
$T$ & the observation time & $N$ & signal length \\
$T_{M}$ & micro-motion/micro-Doppler period & $f_{M}$ & micro-motion/micro-Doppler frequency \\
$F_{x}[\cdot]$ & the Fourier transform of a signal on $x$ variable & $*_{f}$ & convolution in the frequency domain \\
$N_{f}$ & points of the Fourier transform & & \\
\hline
\end{tabular}

makes use of the periodic instantaneous phase, the influence of aperiodic RCS is negligible and this assumption is adopted in the derivation of the proposed method.

The micro-motion modulation component can be rewritten as

$$
s_{M}(t)=a(t) \exp (j \phi(t))
$$

where $a(t)$ and $\phi(t)$ are the instantaneous amplitude and instantaneous phase of $s_{M}(t)$, respectively.

Using (2), (6) and (7), it is not difficult to prove that

$$
\begin{gathered}
a(t)=a\left(t+T_{M}\right) \\
\phi(t)=\phi\left(t+T_{M}\right) \\
s_{M}(t)=s_{M}\left(t+T_{M}\right) .
\end{gathered}
$$

Substituting (3) into (5), the translation modulation component can be rewritten as

$$
s_{T}(t)=\exp \left\{-\frac{j 4 \pi}{\lambda}\left[R_{0}+\sum_{i=1}^{Q} a_{i} t^{i}\right]\right\} .
$$

It is evident that $s_{M}(t)$ is a periodic phase modulated signal (PPMS), $s_{T}(t)$ is a PPS, and $s(t)$ is the combination of a PPMS and a PPS.

The instantaneous Doppler of the $l$ th scatterer is defined as

$$
f_{l}(t)=-\frac{2}{\lambda} \frac{\mathrm{d} r_{l}(t)}{\mathrm{d} t}=f_{T}(t)+f_{M, l}(t)
$$

where

$$
\begin{aligned}
f_{T}(t) & =-\frac{2}{\lambda} \frac{\mathrm{d} r_{T}(t)}{\mathrm{d} t} \\
f_{M, l}(t) & =-\frac{2}{\lambda} \frac{\mathrm{d} r_{M, l}(t)}{\mathrm{d} t} .
\end{aligned}
$$

$f_{T}(t)$ is the translation-Doppler/macro-Doppler of the target, and $f_{M, l}(t)$ is the micro-Doppler of the $l$ th scatterer. The $\mathrm{m}$ $\mathrm{D}$ reflects the micro-motion dynamics of radar target, which is the basis of the m-D signature extraction and parameter estimation. Generally, TFR which provides the time and frequency information simultaneously is employed for $\mathrm{m}-\mathrm{D}$ signature analysis [1]. Note that the $\mathrm{m}-\mathrm{D}$ is circular-shifted by the translation and it causes Doppler-ambiguity when the macro-Doppler exceeds the pulse repetition frequency (PRF) [15]. This makes it difficult to process the m-D signals.

\section{RELATED WORK}

In this section, we overview some existing m-D period estimation methods. These methods have been proved to be effective for $\mathrm{m}-\mathrm{D}$ period estimation and they are widely used in this field.

\section{A. Cyclic spectrum density}

In [11], cyclostationary theory is applied to analyze m-D signal and it is proved that cyclic spectrum density (CSD) can be utilized to estimate the m-D period. The CSD of the radar echo is defined as the Fourier transform of cycloautocorrelation function $R_{s}(\alpha, \tau)$,

$$
\zeta(\alpha, f)=\int_{-\infty}^{\infty} R_{s}(\alpha, \tau) \exp (-j 2 \pi f \tau) \mathrm{d} \tau
$$

where $R_{s}(\alpha, \tau)$ is the Fourier coefficient of the instantaneous autocorrelation function (IAF), $\alpha$ is the cycle frequency, and $\tau$ is the lag,

$$
\begin{gathered}
R_{s}(\alpha, \tau)=\int_{-T / 2}^{T / 2} c_{s}(t, \tau) \exp (-j 2 \pi \alpha t) \mathrm{d} t \\
c_{s}(t, \tau)=s\left(t+\frac{\tau}{2}\right) s^{*}\left(t-\frac{\tau}{2}\right) .
\end{gathered}
$$

It is shown that CSD can be represented as a series of peaks in the $(\alpha, f)$ plane and the amplitudes of these peaks are modulated by the Bessel function. Approximately, the peaks of CSD have the following locations

$$
\left\{\begin{array}{l}
\alpha=(p-q) f_{M} \\
f=\frac{1}{2}(p+q) f_{M}
\end{array} \quad p, q \in Z\right.
$$

The $\alpha$-slice of $\operatorname{CSD} \zeta(\alpha, f=0)$ exhibits peaks at $\alpha=$ $2 i f_{M}, i \in Z$ and this property is utilized for m-D period estimation.

In this work, since $\frac{\tau}{2}$ is difficult to be computed, in the calculation of $R_{s}(\alpha, \tau)$, we refer to another definition of IAF given by

$$
c_{s}(t, \tau)=s(t+\tau) s^{*}(t) .
$$

Then $R_{s}(\alpha, \tau)$ can be rewritten as

$$
R_{s}(\alpha, \tau)=\exp (-j \pi \alpha \tau) \int_{-\frac{T}{2}}^{\frac{T}{2}} c_{s}(t, \tau) \exp (-j 2 \pi \alpha t) \mathrm{d} t .
$$

The calculation of $R_{s}(\alpha, \tau)$ (two-sided $\mathrm{AF}$ and phase multiplication) requires $1 / 2 \cdot(2 N-1) N_{f} \log _{2} N_{f} \quad+$ $N^{2}+2 N N_{f}$ multiplications ${ }^{2} . \quad \zeta(\alpha, f)$ can be obtained by performing a $(2 N-f)$-points Fast Fourier transform (FFT) on $R_{s}(\alpha, \tau)$ along the lag dimension, it requires $1 / 2 \cdot(2 N-1) N_{f} \log _{2}(2 N-1)$ multiplications. The total operations CSD required are

\footnotetext{
${ }^{2}$ Computational complexities of the related signal processing methods are presented in Appendix A.
} 
$1 / 2 \cdot(2 N-1) N_{f}\left(\log _{2}(2 N-1)+\log _{2} N_{f}\right)+N^{2}+2 N N_{f}$ multiplications. Taking $N_{f}=2 N-1$, the computational complexity is $O\left(N^{2} \log _{2} N\right)$ approximately.

\section{B. $2 D$ FFT of $T F$}

The 2D FFT can be used to extract the periodic structure of the spectrogram of m-D signal [9],

$$
\chi\left(f_{x}, f_{y}\right)=\int_{-\infty}^{\infty} \int_{-\infty}^{\infty}\left|\rho_{s}(t, f)\right|^{2} \exp \left(-j 2 \pi t f_{x}-j 2 \pi f f_{y}\right) \mathrm{d} t \mathrm{~d} f
$$

where $\left|\rho_{s}(t, f)\right|^{2}$ is the spectrogram. Collapsed $\chi\left(f_{x}, f_{y}\right)$ in 1D function of frequency by integrating (summing for discrete version) along $f_{y}$ dimension,

$$
\xi\left(f_{x}\right)=\int_{-\infty}^{\infty} \chi\left(f_{x}, f_{y}\right) \mathrm{d} f_{y} .
$$

According to [9], $\xi\left(f_{x}\right)$ shows peaks at $i f_{M}, i \in Z$.

The main computation of this method concentrates on the computation of the spectrogram and the 2D FFT. As $N_{f} \leq N$, the computational complexity is $O\left(N N_{f} \log _{2} N_{f}\right)+$ $N N_{f}\left(\log _{2} N+\log _{2} N_{f}\right) \approx O\left(N N_{f} \log _{2} N\right)$.

\section{C. $T F$ similarity}

The TF similarity proposed in [10] can be expressed as,

$$
M_{s}(m)=\sum_{l=0}^{P-1} \sum_{k=0}^{N_{f}-1} \rho_{s}(l, k) \rho_{s}(m+l, k)
$$

where $\rho_{s}(l, k), 0 \leq l \leq P-1,0 \leq k \leq N_{f}-1$ is a part of the whole TF matrix. From (24), it can be concluded that $M_{s}(m)$ is indeed the 2D correlation coefficient. When the discrete lag $m$ satisfies $m \Delta T \approx i T_{M}, i \in Z$, the correlation coefficients are large, and $M_{s}(m)$ shows peaks at these locations.

The calculation of (24) involves $(N-P+1) P N_{f}$ multiplications. The computational complexity of this method is $O\left(N N_{f} \log _{2} N\right)+(N-P+1) P N_{f}$. As $N N_{f} \leq$ $(N-P+1) P N_{f} \leq 1 / 4 \cdot N(N+2) N_{f}$, the computational complexity lies in $\left[O\left(N N_{f} \log _{2} N\right), O\left(N^{2} N_{f}\right)\right]$.

\section{Circular correlation coefficients}

The circular correlation coefficients is proposed to extract the circular periodicity of the TF in the presence of translation [15]. The circular cross-correlation of two slices of the TF is

$$
C_{r}(k ; n, l)=\sum_{p=0}^{N_{f}-1}\left|\rho_{s}(n, k)\right|\left|\rho_{s}\left(n,(k+p)_{N_{f}}\right)\right| .
$$

The circular correlation coefficients matrix of $\rho_{s}(n, k)$ is defined as

$$
\mathbf{M}_{c}=\left[\begin{array}{cccc}
C_{m}(0,0) & C_{m}(0,1) & \cdots & C_{m}(0, N-1) \\
C_{m}(1,0) & C_{m}(1,1) & \cdots & C_{m}(1, N-1) \\
\vdots & \vdots & \ddots & \vdots \\
C_{m}(N-1,0) & C_{m}(N-1,1) & \cdots & C_{m}(N-1, N-1)
\end{array}\right]
$$

where $C_{m}(n, l)$ is the normalized maximal value of $C_{r}(k ; n, l)$,

$$
C_{m}(n, l)=\frac{\max _{k}\left\{C_{r}(k ; n, l)\right\}}{\sqrt{\sum_{k=0}^{N_{f}-1}\left|\rho_{s}(n, k)\right|^{2}} \sqrt{\sum_{k=0}^{N_{f}-1}\left|\rho_{s}(l, k)\right|^{2}}} .
$$

Define average circular correlation coefficients as the average of the $k$ th diagonal of the matrix

$$
\bar{C}_{r}(k)=\operatorname{mean}\left\{\operatorname{diag}\left(\mathbf{M}_{c}, k\right)\right\}
$$

where

$$
\operatorname{diag}\left(\mathbf{M}_{c}, k\right)=\left\{\mathbf{M}_{c}(n, l), l=n+k, 1 \leq n, l \leq N\right\} .
$$

According to [15], $\bar{C}_{r}(k)$ has the same period with the m-D signal and shows peaks at $k=i N_{T_{M}}, i \in Z$.

The major computation of the circular correlation coefficients concentrates on the computation of $C_{r}(k ; n, l)$ and $P(n, l)=\sqrt{\sum_{k=0}^{N_{f}-1}\left|\rho_{s}(n, k)\right|^{2}} \sqrt{\sum_{k=0}^{N_{f}-1}\left|\rho_{s}(l, k)\right|^{2}}$. The calculation of $C_{r}(k ; n, l)$ can be implemented by a fast algorithm, i.e., an FFT, a conjugated production in the frequency domain, and an inverse FFT, which requires $N_{f} \log _{2} N_{f}+$ $N_{f}$ multiplications. As there are $N(N-1) / 2$ combinations for these slices, the total operations required are $N(N-1)\left(N_{f} \log _{2} N_{f}+N_{f}\right) / 2$ multiplications. In the computation of $P(n, l)$, the terms $\sqrt{\sum_{k=0}^{N_{f}-1}\left|\rho_{s}(n, k)\right|^{2}}, l=$ $0,1, \cdots, N-1$ can be computed and stored at the beginning and the total operations required are $N N_{f}+N(N-1) / 2$. Approximately, the computational complexity of the circular correlation coefficients is $O\left(N^{2} N_{f} \log _{2} N_{f}\right)$.

\section{E. Autocorrelation}

The autocorrelation [5] is the most widely used method for period estimation, which is defined as

$$
r_{s}(m)=\sum_{n=0}^{N-m-1} s(n) s^{*}(n+m) \text {. }
$$

It is easy to derive that the computational complexity of the autocorrelation function is $O\left(N^{2}\right)$.

As mentioned in the introduction, in the presence of translation, all m-D period estimation methods except the circular correlation coefficients should be integrated with a translation compensation step. In this work, the autocorrelation with translation compensation is taken as an example. The translation compensation algorithm adopted here is the minimumspectrum-entropy translation compensation (MSETC) algorithm proposed in [14]. The principle of this algorithm is multiplying the m-D signal by a quadratic phase function that minimize the spectrum entropy. The compensated m-D signal can be expressed as

$$
s_{c}(t)=s(t) \exp \left(j 4 \pi r_{T, c}(t) / \lambda\right)
$$

where $r_{T, c}(t)$ is the quadratic translation used for compensation

$$
r_{T, c}(t)=R_{0, c}+a_{1, c} t+a_{2, c} t^{2}
$$


TABLE II

Computational Complexities of DifFerent M-D Period Estimation Methods

\begin{tabular}{|c|c|c|c|c|c|c|}
\hline AF concentration statistics & CSD & autocorr. & autocorr. + comp. & 2D FFT of TF & TF similarity & circular corr. coeff. \\
\hline$O\left(N^{2} \log _{2} N\right)$ & $O\left(N^{2} \log _{2} N\right)$ & $O\left(N^{2}\right)$ & $O\left(N^{3} \log _{2} N\right)$ & $O\left(N N_{f} \log _{2} N\right)$ & {$\left[O\left(N N_{f} \log _{2} N\right), O\left(N^{2} N_{f}\right)\right]$} & $O\left(N^{2} N_{f} \log _{2} N_{f}\right)$ \\
\hline
\end{tabular}

The objective of compensation is to search the parameters $\theta=$ $\left(R_{0, c}, a_{1, c}, a_{2, c}\right)$ that minimize the spectrum entropy of $s_{c}(t)$. The compensation result is mainly determined by the search range and resolution of $a_{2, c}$. The computation of the MSETC algorithm concentrates on the product of two signal and the spectrum entropy. Assuming $N_{a}$ samples of $a_{2, c}$ are taken, the computational complexity approximates to $O\left(N_{a} N \log _{2} N\right)$.

The searching interval of $a_{2, c}$ is computed according to the parameters of the signal so that the overall range of the residual translation frequency would not exceed the value of a frequency bin $1 / T$,

$$
\delta a=\lambda /\left(2 T^{2}\right) .
$$

Assuming the searching range of $a_{2, c}$ is in the interval $\left[a_{2}-2, a_{2}+2\right]$, which is a quite small range. Thus,

$$
N_{a}=8 T^{2} / \lambda=8 N^{2} \Delta T^{2} / \lambda .
$$

Thus, the computational complexity of the MSETC algorithm is $O\left(N^{3} \log _{2} N\right)$, which is very high in signal processing. And the computational complexity of the autocorrelation with translation compensation approximates to $O\left(N^{3} \log _{2} N\right)$ (The computational complexity of the autocorrelation $O\left(N^{2}\right)$ is negligible compared with this term.). The autocorrelation has the lowest computational complexity compared with other m-D period estimation methods. However, when the autocorrelation is combined with the translation compensation algorithm, the computational complexity increases significantly and has the largest value as shown in Table II.

By summarizing all the aforementioned methods, the estimation statistics in the time/frequency domain (the lag domain is similar to the time domain) has the following properties.

Prop. 1: The statistics in the time domain must present periodic pattern with the same period of the m-D signal, and the statistics in the frequency domain must be derived from a periodic statistics with the same period of the m-D signal.

Prop. 2: To extract the period, the statistics must exhibit peaks at locations that are multiples of the m-D pe$\mathrm{riod} /$ frequency.

\section{AF-BASED M-D PERIOd Estimation}

In radar measurements, the phase (or Doppler) of the radar signal is more stable compared with the RCS. All motionrelated information is embedded in the phase (or Doppler) [1]. Thus, it is preferred to develop the m-D period estimation methods based on the periodic instantaneous phase (or Doppler). It is reported in [18] that the HAF with proper order can transform a PPS into a sinusoidal signal (i.e., a first-order PPS) and the parameters of the signal can be estimated in sequence. Inspired by the HAF, the AF (i.e., first-order HAF) is utilized to extract the m-D period in this work. There are both similarity and difference between the HAF in [18] and the AF adopted here. The similarity is that both the HAF and the AF utilize the spectrum of the phase difference signal to estimate the parameters, while the difference is that the considered signal in this work has a complicated modulation form, i.e., the combination of a PPS and a PPMS.

Since our approach attempts to measure the phase periodicity in the time domain, it must be consistent with Prop. 1 and Prop. 2. In this section, the details of the AF concentration statistics for the micro-motion modulation component (the MM case) is presented firstly, and then the influence of translation (the MMLT case and the MMQT case) is given.

\section{A. AF Concentration Statistics of the Micro-Motion Modula- tion Component}

The ambiguity function of $s_{M}(t)$ is defined as,

$$
C_{s_{M}}(f, \tau)=\int_{0}^{T-\tau} c_{s_{M}}(t, \tau) \exp (-j 2 \pi f t) d t
$$

where $c_{s_{M}}(t, \tau)$ is the IAF of $s_{M}(t)$,

$$
c_{s_{M}}(t, \tau)=s_{M}(t) s_{M}^{*}(t+\tau) .
$$

$\tau$ is the lag, $0 \leq \tau \leq T, 0 \leq t \leq T-\tau ; T$ is the observation time. For simplicity, in the following derivation, an infinite observation time (i.e., $T \rightarrow \infty$ ) is considered firstly, and the effect of the finite observation time is discussed next.

\section{i). Infinite observation time.}

Denote $c_{s_{M}}^{\infty}(t, \tau)$ as the IAF with infinite observation time, $c_{s_{M}}(t, \tau)$ can be reformulated as

$$
c_{s_{M}}(t, \tau)=c_{s_{M}}^{\infty}(t, \tau) \operatorname{rect}\left(\frac{t-(T-\tau)}{T-\tau}\right) .
$$

Substituting (8) into (36)

$$
c_{s_{M}}^{\infty}(t, \tau)=a(t) a^{*}(t+\tau) \exp (j \phi(t)-j \phi(t+\tau)) .
$$

To analyze the periodicity, letting $\tau=\tau+T_{M}$, the following formula is obtained as

$$
\begin{aligned}
& c_{s_{M}}^{\infty}\left(t, \tau+T_{M}\right) \\
& =a(t) a^{*}\left(t+\tau+T_{M}\right) \exp \left(j \phi(t)-j \phi\left(t+\tau+T_{M}\right)\right) \\
& =a(t) a^{*}(t+\tau) \exp (j \phi(t)-j \phi(t+\tau)) \\
& =c_{s_{M}}^{\infty}(t, \tau) .
\end{aligned}
$$

Similarly, letting $t=t+T_{M}$, we have

$$
c_{s_{M}}^{\infty}\left(t+T_{M}, \tau\right)=c_{s_{M}}^{\infty}(t, \tau) .
$$

Therefore, the IAF is a periodic function with respect to both the time and lag. By taking a Fourier transform along the time dimension, we get a lag-periodic ambiguity function,

$$
C_{s_{M}}^{\infty}\left(f, \tau+T_{M}\right)=C_{s_{M}}^{\infty}(f, \tau)
$$




$$
\left|C_{s_{M}}^{\infty}\left(f, \tau+T_{M}\right)\right|=\left|C_{s_{M}}^{\infty}(f, \tau)\right| .
$$

We now discuss the relation between the AF and the instantaneous phase and more insight into why the AF concentration statistics is used can be obtained from this discussion. (38) can be rewritten as

$$
c_{s_{M}}^{\infty}(t, \tau)=A(t, \tau) \exp (j \psi(t, \tau))
$$

where $A(t, \tau)$ and $\exp (j \psi(t, \tau))$ are the amplitude correlation component and the phase difference component, respectively.

$$
\begin{gathered}
A(t, \tau)=a(t) a^{*}(t+\tau) \\
\exp (j \psi(t, \tau))=\exp (j \phi(t)-j \phi(t+\tau)) .
\end{gathered}
$$

According to the property of Fourier transform, $C_{s_{M}}^{\infty}(f, \tau)$ can be expressed as

$$
C_{s_{M}}^{\infty}(f, \tau)=F_{t}[A(t, \tau)] *_{f} F_{t}[\exp (j \psi(t, \tau))] .
$$

The amplitude $a(t)$ varies more slowly compared with the instantaneous phase $\phi(t)$ (because the phase is more sensitive to the range variation induced by micro-motion dynamics). It is expected that the shape of the spectrum $C_{s_{M}}^{\infty}(f, \tau)$ is mainly determined by the phase difference component. $C_{s_{M}}^{\infty}(f, \tau)$ will concentrate at near zero frequency when the phase difference is small. Conversely, $C_{s_{M}}^{\infty}(f, \tau)$ will spread over a wide frequency range when the phase difference is large. Therefore, a concentration measure of $C_{s_{M}}^{\infty}(f, \tau)$ can characterize the periodic change of the phase difference. Moreover, the concentration measure would present peaks when the phase difference is zero.

Herein, the entropy [19] is employed as a concentration measure to demonstrate the aforementioned properties. The entropy of $C_{s_{M}}^{\infty}(f, \tau)$ along the frequency dimension, referred to as $\mathrm{AF}$ entropy, is given by

$$
\varepsilon_{s_{M}}^{\infty}(\tau)=\int_{-\infty}^{\infty} \frac{C_{s_{M}}^{\infty}(f, \tau)}{E_{C_{s_{M}}^{\infty}}(\tau)} \ln \frac{C_{s_{M}}^{\infty}(f, \tau)}{E_{C_{s_{M}}^{\infty}}(\tau)} d f
$$

where

$$
E_{C_{s_{M}}^{\infty}}(\tau)=\int_{-\infty}^{\infty}\left|C_{s_{M}}^{\infty}(f, \tau)\right| d f
$$

is the energy of $C_{s_{M}}^{\infty}(f, \tau)$ at slice $\tau$. Substituting (42) into (47) and (48), it is easy to derive that

$$
\varepsilon_{s_{M}}^{\infty}\left(\tau+T_{M}\right)=\varepsilon_{s_{M}}^{\infty}(\tau) .
$$

Thus, the AF entropy has the same periodicity with the micromotion dynamics and Prop. 1 is satisfied.

Now Prop. 2 is considered. When $\tau=i T_{M}$, the phase difference component $\exp (j \psi(t, \tau))$ is equivalent to 1 as

$$
\exp \left(j \psi\left(t, i T_{m}\right)\right)=\exp \left(j \phi(t)-j \phi\left(t+i T_{M}\right)\right)=1 .
$$

We have

$$
F\left[\exp \left(j \psi\left(t, i T_{m}\right)\right)\right]=\delta(f) .
$$

where $\delta(f)$ is the Dirac delta function. It is clear that $\varepsilon_{s_{M}}^{\infty}\left(i T_{M}\right)$ shows peaks at $\tau=i T_{M}$. While for other lags $\tau \neq i T_{M}$, the values of $\varepsilon_{s_{M}}^{\infty}\left(i T_{M}\right)$ are small. This property can be expressed as

$$
\varepsilon_{s_{M}}^{\infty}(\tau) \leq \varepsilon_{s_{M}}^{\infty}\left(i T_{M}\right), \quad i T_{M} \leq \tau \leq(i+1) T_{M} .
$$

From the above analysis, it is proved that the AF entropy satisfies Prop. 1 and Prop. 2, thus it can be used as an m-D period estimator.

\section{ii). Finite observation time.}

In this subsection, we will discuss the situation where the observation time is finite. In this situation, the time-support of the IAF is a function with respect to the lag. The effect of the varying time-support of the IAF is modelled and new definitions of AF entropy is presented.

Substituting $\tau=\tau_{0}$ and $\tau=\tau+i T_{M}$ into (37), we have

$$
\begin{gathered}
c_{s_{M}}\left(t, \tau_{0}\right)=c_{s_{M}}^{\infty}\left(t, \tau_{0}\right) \operatorname{rect}\left(\frac{t-\left(T-\tau_{0}\right) / 2}{T-\tau_{0}}\right) \\
c_{s_{M}}\left(t, \tau_{0}+i T_{M}\right)=c_{s_{M}}^{\infty}\left(t, \tau_{0}\right) \operatorname{rect}\left(\frac{t-\left(T-\tau_{0}+i T_{M}\right) / 2}{T-\tau_{0}+i T_{M}}\right)
\end{gathered}
$$

where $0 \leq \tau_{0}<T_{M}, 0 \leq \tau_{0}+i T_{M}<T$. Then,

$$
\begin{gathered}
C_{s_{M}}\left(f, \tau_{0}\right)=C_{s_{M}}^{\infty}\left(f, \tau_{0}\right) *_{f}\left[\left(T-\tau_{0}\right)\right. \\
\left.\cdot \exp \left(-j \pi f\left(T-\tau_{0}\right)\right) \operatorname{sinc}\left(\left(T-\tau_{0}\right) f\right)\right] \\
C_{s_{M}}\left(f, \tau_{0}+i T_{M}\right)=C_{s_{M}}^{\infty}\left(f, \tau_{0}\right) *_{f}\left[\left(T-\tau_{0}+i T_{M}\right)\right. \\
\left.\cdot \exp \left(-j \pi f\left(T-\tau_{0}+i T_{M}\right)\right) \operatorname{sinc}\left(\left(T-\tau_{0}+i T_{M}\right) f\right)\right] .
\end{gathered}
$$

Since the convolution with the sinc function acts as an interpolation operator in signal processing, $\left|C_{s_{M}}\left(f, \tau_{0}+i T_{M}\right)\right|$ can be viewed as the resampling of $\left|C_{s_{M}}\left(f, \tau_{0}\right)\right|$ with an amplitude scale. Thus, we have the following approximation

$$
\left|C_{s_{M}}\left(T_{s}^{i} f, \tau_{0}+i T_{M}\right)\right| \approx T_{s}^{i}\left|C_{s_{M}}\left(f, \tau_{0}\right)\right|
$$

where

$$
T_{s}^{i}=\frac{T-\left(\tau_{0}+i T_{M}\right)}{T-\tau_{0}}
$$

is the time-support scale.

A redefined AF entropy considering the varying time support is given as follows and more details are given in Appendix B.

$$
\varepsilon_{s_{M}}(\tau)=\int_{-\infty}^{\infty} \frac{C_{s_{M}}(f, \tau)}{E_{C_{s_{M}}}(\tau)} \ln \frac{C_{s_{M}}(f, \tau)}{E_{{S_{s_{M}}}(\tau)}} d f-\ln (T-\tau) .
$$

It is easy to prove that $\varepsilon_{s_{M}}(\tau)$ satisfies the two aforementioned properties approximately.

\section{B. The Influence of Translation on AF Concentration Statistics}

For targets with translation, the translation modulation component $s_{T}(t)$ is a PPS. As mentioned in [18], the phase differentiation (PD) operation in the AF can reduce the order of polynomial-phase signals. Thus, the AF has the potential to process $\mathrm{m}$-D signals in the presence of translation. For simplicity, in this subsection, we neglect the varying timesupport of the IAF. As the effect of varying time-support is considered in the modified AF concentration statistics, this simplification would not affect the conclusion.

According to (4), the IAF of the radar echo can be expressed as

$$
\begin{aligned}
c_{s}(t, \tau) & =s_{T}(t) s_{T}^{*}(t+\tau) s_{M}(t) s_{M}^{*}(t+\tau) \\
& =c_{s_{T}}(t, \tau) c_{s_{M}}(t, \tau)
\end{aligned}
$$


where

$$
c_{s_{T}}(t, \tau)=\exp \left\{-\frac{j 4 \pi}{\lambda}\left[\sum_{i=1}^{Q} a_{i} t^{i}-\sum_{i=1}^{Q} a_{i}(t+\tau)^{i}\right]\right\}
$$

is the IAF of the translation modulation component.

Now the effect of the translation on the AF and the AF concentration statistics is considered.

\section{i). The MMLT case.}

Substituting $Q=1$ into (61), we have

$$
\begin{gathered}
c_{s_{T}}(t, \tau)=\exp \left\{\frac{j 4 \pi}{\lambda} a_{1} \tau\right\} \\
c_{s}(t, \tau)=\exp \left\{\frac{j 4 \pi}{\lambda} a_{1} \tau\right\} c_{s_{M}}(t, \tau) .
\end{gathered}
$$

For a fixed lag $\tau, c_{s_{T}}(t, \tau)$ is a constant phase with respect to $t$. Using the linear property of Fourier transform, it is clear that

$$
C_{s}(f, \tau)=\exp \left\{\frac{j 4 \pi}{\lambda} a_{1} \tau\right\} C_{s_{M}}(f, \tau) .
$$

The constant term $\exp \left\{\frac{j 4 \pi}{\lambda} a_{1} \tau\right\}$ in (64) has no influence on the pattern and the concentration behavior of $C_{s}(f, \tau)$, thus the entropy of $C_{s}(f, \tau)$ would be the same as $C_{s_{M}}(f, \tau)$.

$$
\varepsilon_{s}(\tau)=\varepsilon_{s_{M}}(\tau) .
$$

This property can be easily proved by substituting $\left|C_{s}(f, \tau)\right|=$ $\left|C_{s_{M}}(f, \tau)\right|$ into (47).

\section{ii). The MMQT case.}

Substituting $Q=2$ into (61), we have

$$
c_{s_{T}}(t, \tau)=\exp \left\{\frac{j 4 \pi}{\lambda}\left(a_{1} \tau+a_{2} \tau^{2}+2 a_{2} \tau t\right)\right\}
$$

and

$$
C_{s_{T}}(f, \tau)=\exp \left\{\frac{j 4 \pi}{\lambda}\left(a_{1} \tau+a_{2} \tau^{2}\right)\right\} \delta\left(f-\frac{4 a_{2} \tau}{\lambda}\right) .
$$

Note that, in the calculation of $C_{s_{T}}(t, \tau)$, an infinite observation time is considered. Otherwise the $\delta(\cdot)$ function in (67) would be replaced by sinc $(\cdot)$ function.

Therefore,

$$
\begin{aligned}
C_{s}(f, \tau) & =C_{s_{T}}(f, \tau) *_{f} C_{s_{M}}(f, \tau) \\
& =\exp \left\{\frac{j 4 \pi}{\lambda}\left(a_{1} \tau+a_{2} \tau^{2}\right)\right\} C_{s_{M}}\left(f-\frac{4 a_{2} \tau}{\lambda}, \tau\right) .
\end{aligned}
$$

It is clear that from (68), $C_{s}(f, \tau)$ is the frequency-shifted version of $C_{s_{M}}(f, \tau)$ with a constant phase (For the discrete signal, the frequency-shift is replaced by the circularfrequency-shift). This frequency shift makes $C_{s}(f, \tau)$ more complicated. Fortunately, the entropy of a signal is merely determined by its energy distribution, thus the entropy of $C_{s}(f, \tau)$ is also the same as $C_{s_{M}}(f, \tau)$ in this case.

Using $\left|C_{s}(f, \tau)\right|^{2}=\left|C_{s_{M}}\left(f-\frac{4 a_{2} \tau}{\lambda}, \tau\right)\right|^{2}, E_{C_{s}}(\tau)=$ $E_{C_{s_{M}}}(\tau)$, followed by an substituting of $f^{\prime}=f-\frac{4 a_{2} \tau}{\lambda}$, it can be proved that

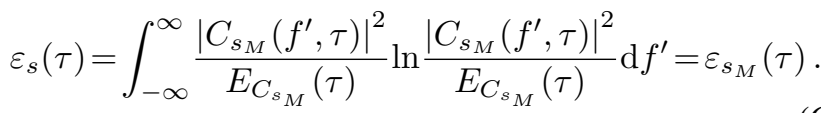

From the above derivation in Subsection A and B, the following conclusions can be made: (i). The AF of the micromotion modulation component presents the same periodicity with the micro-motion; (ii). The periodicity is replaced by the circular periodicity in the presence of translation; (iii). The AF entropy of the radar echo is (or approximately) invariant to translation with polynomial terms not larger than second order; (iv). The AF concentration statistics show peaks at lags which are multiples of the m-D period and can be used to estimate the m-D period.

\section{The Discrete Version and its Implementation}

The discrete versions of AF concentration statistics are essential for implementation. Considering a discrete signal $s(n)$, the discrete IAF of the signal is defined as follows,

$$
c_{s}(n, m)=s(n) s^{*}(n+m)
$$

where $m$ is the discrete lag, $0 \leq m \leq N-1,0 \leq n \leq$ $N-m+1$, and $N$ is the signal length.

The discrete AF of the signal can be obtained by performing a $N_{f}$-points discrete Fourier transform on $c_{s}(n, m)$, which is given by

$$
C_{s}(k, m)=\sum_{n=0}^{N-m-1} c_{s}(n, m) \exp \left(-j 2 \pi n k / N_{f}\right)
$$

where $k=0,1, \cdots, N_{f}-1$. Neglecting an amplitude scale, $C_{s}(k, m)$ can be viewed as the sampling of $C_{s}(f, \tau)$ with a frequency interval $\Delta f=f_{s} / N_{f}$.

The integral of a function $g(f)$ over an interval $[a, b]$ can be approximated by

$$
\int_{a}^{b} g(f) \approx \sum_{k=0}^{K-1} g\left(f_{k}\right) \Delta f
$$

where $f_{k}=a+k \Delta f$ and $\Delta f=(b-a) / K$ are the sampling point and the sampling interval, respectively. Using this property, (59) can be approximated as

$$
\begin{aligned}
\varepsilon_{s}(\tau) \approx \varepsilon_{s}(m) & =\sum_{k=0}^{N_{f}-1} \frac{\left|C_{s}(k, m)\right|^{2}}{E_{C_{s}}(m)} \ln \frac{\left|C_{s}(k, m)\right|^{2}}{E_{C_{s}}(m)} \\
& -\ln \left(\frac{f_{s}}{N_{f}}\right)-\ln \left(\frac{N-m}{f_{s}}\right)
\end{aligned}
$$

where

$$
E_{C_{s}}(m)=\sum_{k=0}^{N_{f}-1}\left|C_{s}(k, m)\right|^{2} .
$$

In practice, we find that if the lag is close to the signal length, the length of the discrete IAF is too short and the concentration statistics tends to be exaggerated. It is essential to include a length-dependent penalize term. By removing the last two terms in (73), the discrete version of AF entropy is redefined as

$$
\varepsilon_{s}(m)=\sum_{k=0}^{N_{f}-1} \frac{\left|C_{s}(k, m)\right|^{2}}{E_{C_{s}}(m)} \ln \frac{\left|C_{s}(k, m)\right|^{2}}{E_{C_{s}}(m)} .
$$

Fig. 1 shows the flowchart of the AF entropy-based estimation approach. In the proposed approach, the entropy can be 


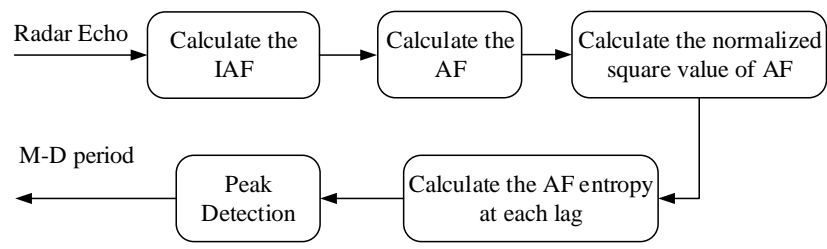

Fig. 1. Flowchart of AF entropy-based m-D period estimation.

replaced by other concentration statistics. One is the ratio of $l_{4}$ norm to the square of $l_{2}$ norm of AF, denoted as AF $l_{4}-l_{2}$ norm (see Appendix A for the continuous version),

$$
\eta_{s}(m)=\sum_{k=0}^{N_{f}-1}\left|C_{s}(k, m)\right|^{4} /\left(\sum_{k=0}^{N_{f}-1}\left|C_{s}(k, m)\right|^{2}\right)^{2} .
$$

The other one is the normalized $l_{1}$ norm of AF, denoted as AF $l_{1}$ norm

$$
\kappa_{s}(m)=\sum_{k=0}^{N_{f}-1}\left|C_{s}(k, m)\right| / \max _{k}\left|C_{s}(k, m)\right| .
$$

The computation of the AF concentration statistics involves the computation of the AF and the computation of the concentration statistics. The computational complexities of these three AF concentration statistics are $O\left(N^{2} \log _{2} N\right)+N \cdot O(N)=$ $O\left(N^{2} \log _{2} N\right)$ (More details can be found in Appendix A).

The computational complexities of all the methods are summarized in Table II. Note that autocorr., autocorr. + comp., and circular corr. coeff. are short notations for autocorrelation, autocorrelation with translation compensation, and circular correlation coefficients, respectively. From Table II, it can be known that the three AF concentration statistics have medium computational cost. While autocorrelation with compensation and circular correlation coefficients are the two most computational expensive algorithms. The autocorrelation has the least computational cost. Moreover, among all the translationcompensation-free $\mathrm{m}$-D period estimation methods, the three $\mathrm{AF}$ concentration statistics have the least computational cost.

\section{DATA VERIFICATION}

\section{A. A Coning Target}

The ballistic missile (BM) is one of the well-studied radar targets with micro-motion. In order to maintain the attitude (orientation), besides the bulk motion, the BM usually undergoes coning motion. The cone target with coning motion can be used to model the BM [9]. The target model is shown in Fig. 2. There are three scatterers on the target with positions $P_{1}=(0,0,1.6) \mathrm{m}, P_{2}=(0,-0.2,-0.4) \mathrm{m}$ and $P_{3}=(0,-0.15,0.1) \mathrm{m}$ in the local coordinate system $O-x y z$, while their RCS are $\sigma_{1}=1, \sigma_{2}=0.5$ and $\sigma_{3}=0.3$. The target is coning with period $T_{M}=2 \mathrm{~s}$, and coning angle $\theta=15^{\circ}$. The azimuth and elevation angle of the target center with respect to the radar in the reference coordinate system are $\alpha=-50^{\circ}$ and $\nu=270^{\circ}$, respectively. The radar operates at $10 \mathrm{GHz}$ with a PRF of $200 \mathrm{~Hz}$. The observation time is $T=4 \mathrm{~s}$. The range of the linear translation and the quadratic translation are
$r_{T}(t)=-t$ and $r_{T}(t)=-t+0.4 t^{2}$, respectively. Fig. 3 shows the flowchart of the coning motion simulation. Following the similar derivation in [1], the range of scatterers corresponding to the micro-motion can be calculated as

$$
\begin{aligned}
& r(t)=\sin \alpha(y \sin \theta+z \cos \theta) \\
& +\cos 2 \pi f_{M} t \cos \alpha(x \cos \nu+y \sin \nu \cos \theta-z \sin \nu \sin \theta) \\
& +\sin 2 \pi f_{M} t \cos \alpha(x \sin \nu-y \cos \nu \cos \theta+z \cos \nu \sin \theta)
\end{aligned}
$$

where $(x, y, z)$ are the coordinates of the scatterers. The micromotion modulation component and the translation component are generated by using (6) and (5), respectively.

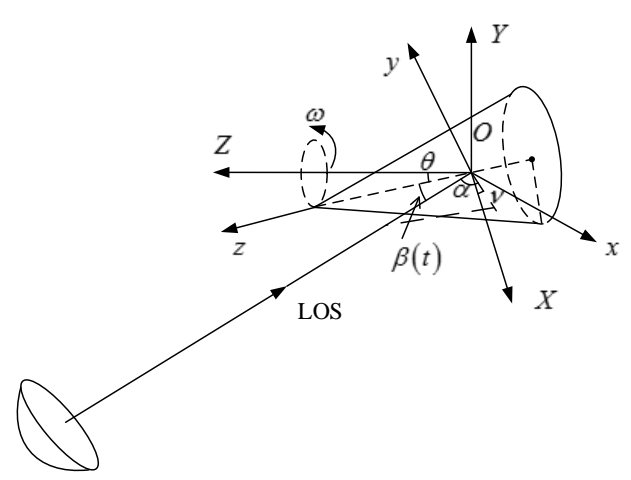

Fig. 2. The cone target model.

In the coning target simulation, the SNR is fixed at $2 \mathrm{~dB}$ except the Monte Carlo simulation. The SNR is defined as $\mathrm{SNR}=10 \log 10 \frac{\sum_{n=0}^{N-1}|s(n)|^{2}}{N \sigma_{v}^{2}}$, where $s(n), n=0,1, \cdots, N-$ 1 is the discrete radar echo, $\sigma_{v}^{2}$ is the variance of the noise.

In Fig. 4, the STFT with a 65-length hamming window is performed on the radar echo to demonstrate the Doppler variation of the target. For the MM case, the coning target exhibits sinusoidal curves in the TF domain which means that the radar echo is sinusoidal-frequency modulated. It is also clear that the STFT of the radar echo is periodic along the time dimension. For the MMLT case and MMQT case, the STFT is the circular-frequency (Doppler)-shifted version of the MM case [15]. Specifically, the Doppler shift value is constant for the MMLT case. While for the MMQT case, the signal is Doppler ambiguous and the Doppler shift value is a wrapped linear function with respect to time.

Fig. 5 plots the AFs of the radar echoes. The AF is normalized along the Doppler dimension by its maximum absolute value at each lag. It is clear that the AFs of radar echoes are the same for the MM case and the MMLT case as mentioned in Section III. While the AF of the radar echo for the MMQT case is the phase-multiplied and circular-Doppler-shifted version of the MM case. A comparison can be made between the AF and the STFT. First, the circular-Doppler-shift effect of the TF appears when there are translation with polynomial terms not less than first order, while this effect of the AF appears only for translation with polynomial terms not less than second order (because the AF can reduce the order of a PPS by one). Second, the line trends of the TF and the AF have contrary directions for the MMQT case. The slices of AF of the radar echo for 


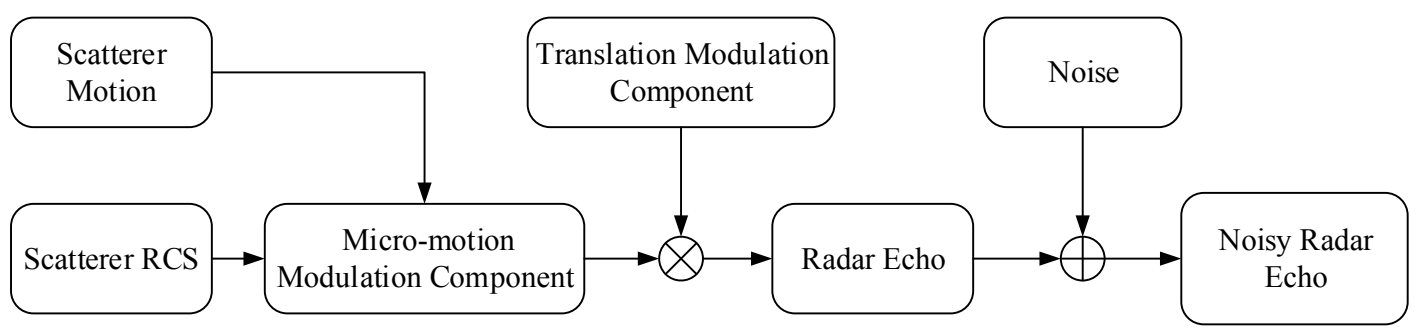

Fig. 3. Flowchart of the coning motion simulation.

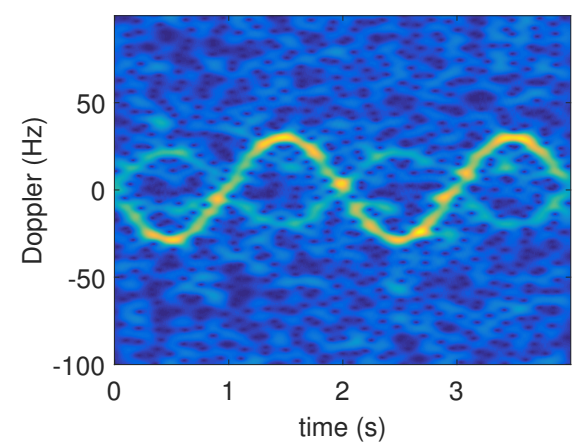

(a)

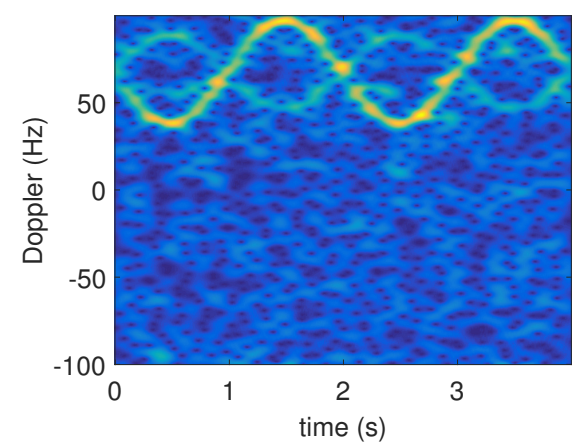

(b)

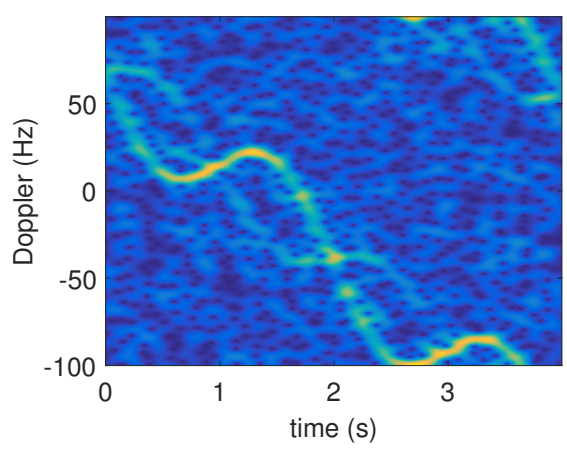

(c)

Fig. 4. STFT of the radar echo.(a) the MM case; (b) the MMLT case; (c) the MMQT case.

the MM case is also displayed in Fig. 5(d)-(f) to examine the concentration effect of the AF. All the AFs of radar echoes concentrate at zero frequency and exhibit good concentration at lags (e.g., $\tau=0,2 \mathrm{~s}$ ) which are multiples of the $\mathrm{m}-\mathrm{D}$ period, while poor concentration appears at other lags. This effect is consistent with the analysis in Section III. A and B.

Next, Fig. 6 demonstrates the estimation statistics of the m-D estimation approaches. The m-D period estimates (based on the distance of the peaks) are summarized in Table III. Estimates close to $2.0 \mathrm{~s}$ are successful estimates while "na" means that the approach fails to provide an m-D period estimate. As most of the estimation statistics (except for the CSD slice) of the MMLT case are the same with that of the MM case, the results of the MM case are not shown.

Fig. 6(a) - (c) show the results of the AF entropy, the AF $l_{4}-l_{2}$ norm and the AF $l_{1}$ norm. As the AF concentration statistics can inherently remove the translation effect, these three AF concentration statistics are approximately the same for the MMLT case and the MMQT case, which can provide good estimates in the presence of the translation.

Fig. 6(d) plots the TF entropy for a comparison. Though the overall concentration of the TF is much better compared with $\mathrm{AF}$, the TF entropy does not have any physical meaning and it is not periodic. Thus, it is not suitable for the m-D period estimation.

Fig. 6(e) presents the result of another AF-relevant approach, the cyclic spectrum density (CSD). It is observed that the slice of CSD of the radar echo without translation exhibits peaks at $\alpha=i f_{M}$, which agrees with the conclusion in [11]. The peaks come from the periodic texture of the AF. Since the AF is spike-like, the quality of the slice of CSD is not good enough and exists harmonic frequencies with disordered amplitudes (it is quite different from that of the radar echo of a single scatterer presented in [11].) This property makes it difficult to estimate the m-D period. What's worse, the slices of CSD for the MMLT case and MMQT case act as a random function and do not have any periodicity. Thus, the application of the CSD in the presence of translation is limited.

Fig. 6(f) shows the result of autocorrelation. The autocorrelations of the m-D signal for the MMLT case exhibits peak at $\tau=2 \mathrm{~s}$. While for the MMQT case, there is no peak at $\tau=2 \mathrm{~s}$, which means that the autocorrelation fails to provide an estimate. To further evaluate the performance of the autocorrelation in the presence of translation by integrating a translation compensation step, the minimum-spectrum-entropy translation compensation algorithm [14] with different searching acceleration resolutions (i.e, $\delta a, 2 \delta a$, and $8 \delta a$ ) is utilized. It is observed that the effectiveness of the new combined method depends on the accuracy of the translation compensation. When the translation compensation is not accurate enough (in this case, with a searching acceleration resolution no less than $8 \delta a$ ), the estimation is unsuccessful. The estimates for cases with $\delta a$ and $2 \delta a$ are $2.0 \mathrm{~s}$ and $2.005 \mathrm{~s}$, respectively.

Fig. $6(\mathrm{~g})$ shows the sum of the 2D FFT of the TF along the frequency dimension. The 2D FFT of TF of the MMLT case presents peaks at $i f_{M}$, while that of MMQT case does not have the property. It is clear that the 2D FFT of TF can only work for the MM case and the MMLT case. The rationale behind this is that the 2D FFT can only extract texture periodicity or constant-wrapped texture periodicity for TF images. However, the quadratic translation introduces time-varying wrapping (time-varying circular Doppler/frequency shift) effect in the TF domain, making the 2D FFT invalid for the m-D period estimation for the MMQT case. 


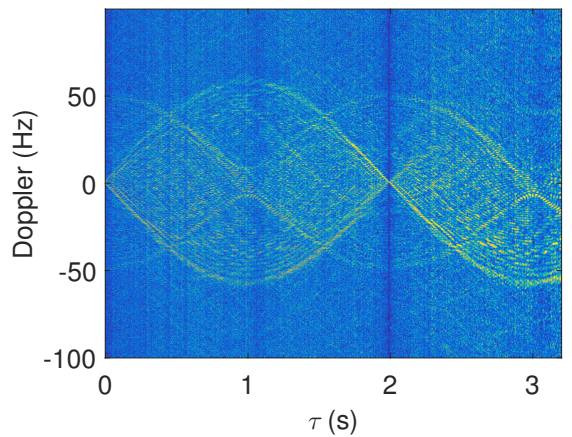

(a)

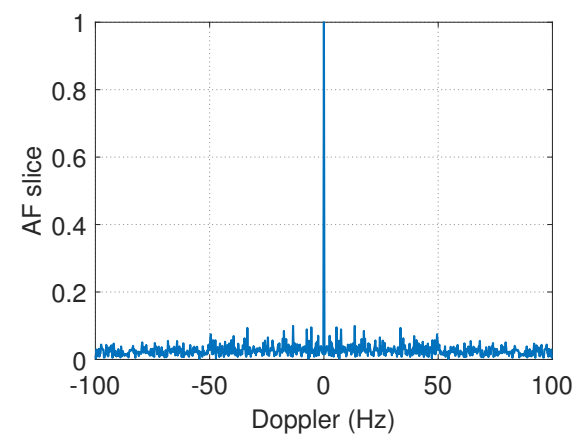

(d)

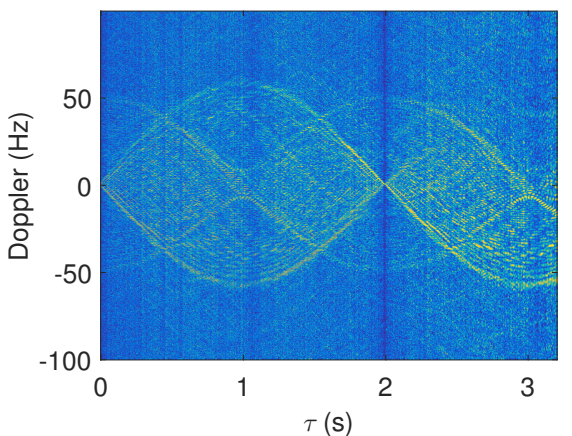

(b)

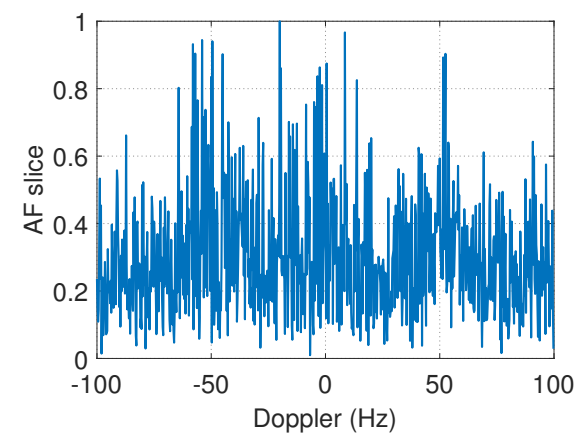

(e)

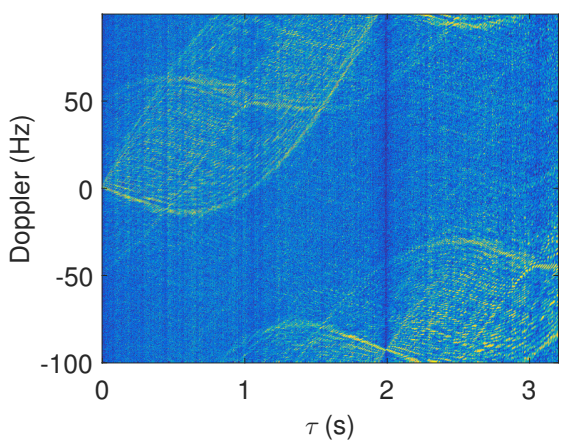

(c)

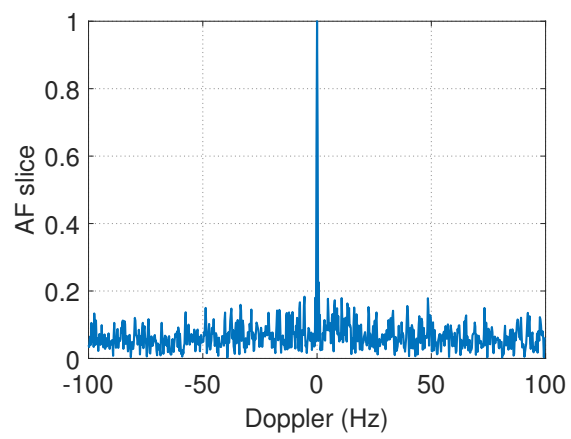

(f)

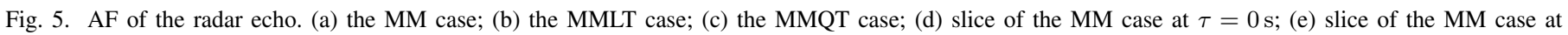
$\tau=1 \mathrm{~s}$; (f) slice of the MM case at $\tau=2 \mathrm{~s}$.

TABLE III

M-D Period Estimates ( $\mathrm{S}$ ) OF The Coning TARget

\begin{tabular}{ccccccccc}
\hline \hline cone example & $\mathrm{AF}$ entropy & $\mathrm{AF} l_{4}-l_{2}$ norm & $\mathrm{AF} l_{1}$ norm & autocorr. & autocorr. + comp. & 2D FFT of TF & TF similarity & circular corr. coeff \\
\hline MMLT & 2.0 & 2.0 & 2.0 & 2.0 & - & 2.286 & 2.005 & 1.995 \\
\hline MMQT & 2.0 & 2.0 & 2.0 & na & $2.0 / 2.005 /$ na $^{a}$ & na & na & 2.0 \\
\hline
\end{tabular}

${ }^{a}$ These three values represent three estimates with different searching acceleration resolutions $\delta a, 2 \delta a$ and $8 \delta a$, respectively.

TABLE IV

MINIMUM SNR (dB) REQUIRED FOR EFFECTIVE ESTIMATES.

\begin{tabular}{ccccccccc}
\hline \hline cone example & $\mathrm{AF}$ entropy & $\mathrm{AF} l_{4}-l_{2}$ norm & $\mathrm{AF} l_{1}$ norm & autocorr. & autocorr. + comp. & 2D FFT of TF & TF similarity & circular corr. coeff \\
\hline MMLT & -2 & -3 & -4 & -2 & - & 10 & 3 & 0 \\
\hline MMQT & -3 & -4 & -4 & na & -2 & na & na & -1 \\
\hline
\end{tabular}

The TF similarity, which exploits the TF periodicity, can be utilized as well. It can be viewed as 2D correlation of TF. Fig. 6(h) shows the results. Similar with the 2D FFT of TF, the TF similarity only works for the MM case and MMLT case.

The results of the circular correlation coefficients, which explicitly exploits the circular periodicity of the TFR for the first time, is presented in Fig. 6(i) for a comparison. It is clear that the circular correlation coefficients for the MMQT case are almost the same with that of the MMLT case (if the small ripples are neglected). Despite of the finite observation time effect, the circular correlation coefficient is invariant to the translation [15]. The shortcoming of the circular correlation coefficients is its less computational efficiency.

Additionally, Monte Carlo simulations (500 realizations) are carried out to evaluate the performance of the aforementioned approaches (the CSD is excluded as it is difficult to get the estimation in this example) in the conditions of different SNRs. The SNR of the signal varies from $-5 \mathrm{~dB}$ to $15 \mathrm{~dB}$ with an interval of $1 \mathrm{~dB}$. Simulation results are plotted in Fig. 7. The Bias and the RMSE (root mean square error) are used as the performance metrics. The Bias and the RMSE are defined as

$$
\operatorname{Bias}=1 / I \sum_{i=1}^{i=I}\left(\hat{T}_{i}-T_{M}\right)
$$

and

$$
\operatorname{RMSE}=\sqrt{\sum_{i=1}^{i=I}\left(\hat{T}_{i}-T_{M}\right)^{2} / I}
$$




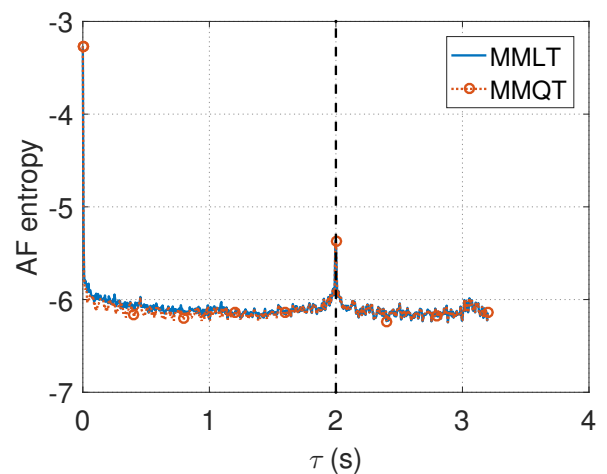

(a)

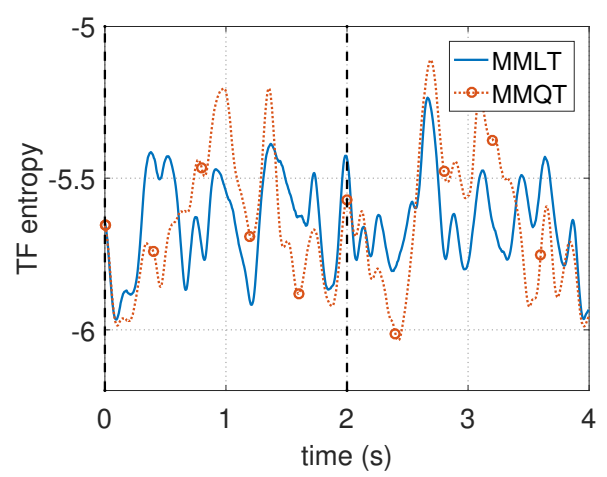

(d)

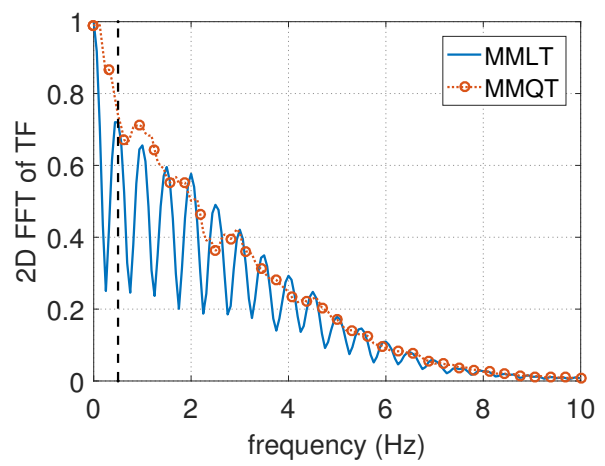

(g)

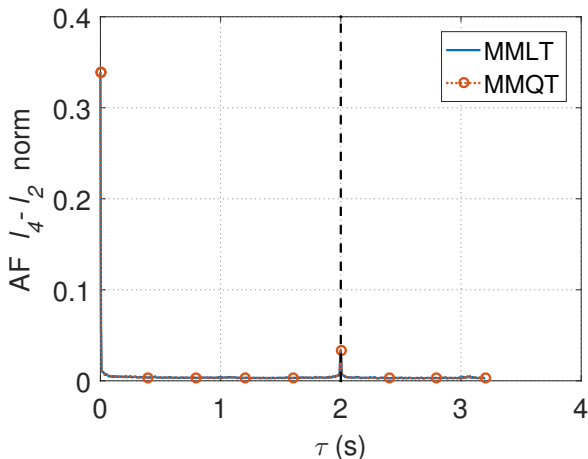

(b)

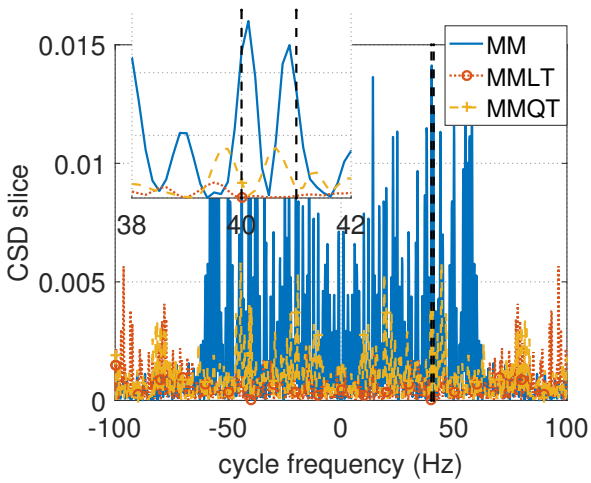

(e)

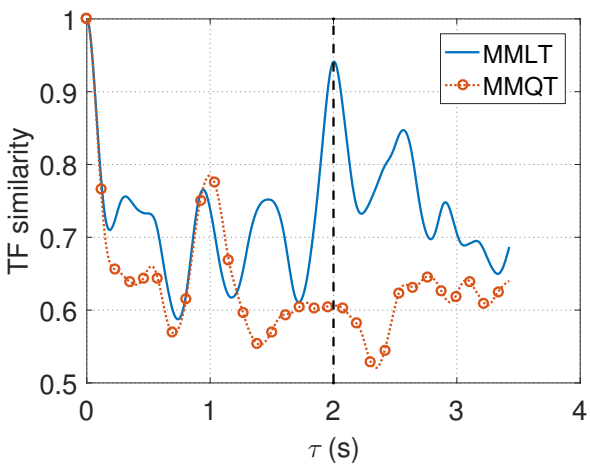

(h)

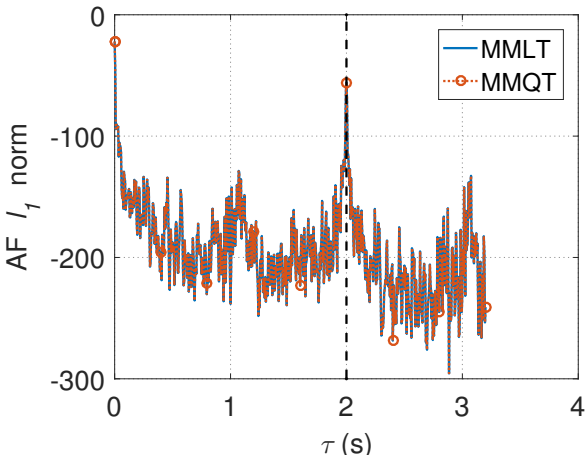

(c)

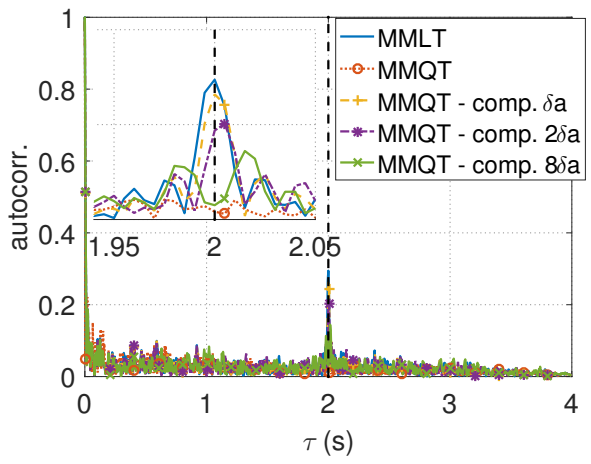

(f)

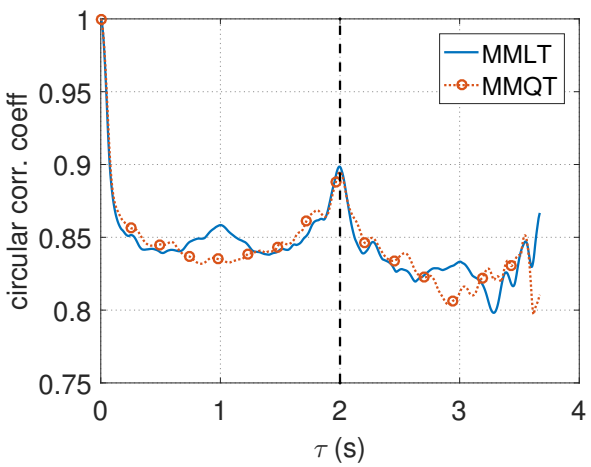

(i)

Fig. 6. Estimation Statistics. (a) AF entropy; (b) AF $l_{4}-l_{2}$ norm; (c) AF $l_{1}$ norm; (d) TF entropy; (e) slices of CSD; (f) autocorrelation (the dash-dot lines with circle, square and asterisk markers denote the results by integrating translation compensation with different search intervals); (g) 2D FFT of TF; (h) TF similarity; (i) circular correlation coefficients. The true m-D period/frequency is indicated by a black dashed vertical line in (a), (b), (c), (f), (g), (h), (i). While in (d) and (e), two vertical lines with distance of the true m-D period and two multiples of the m-D frequency are plotted which means that the statistics should have periodic peaks or periodic pattern at these two instants if they are effective.

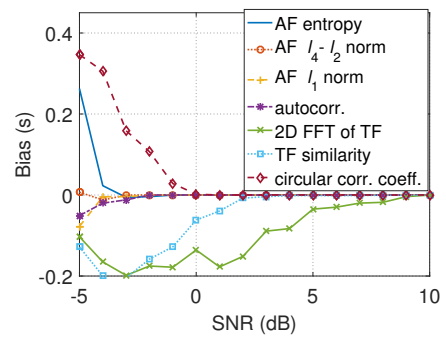

(a)

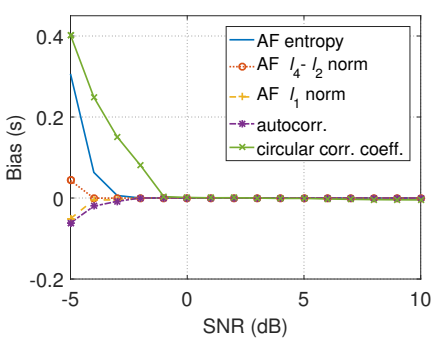

(b)

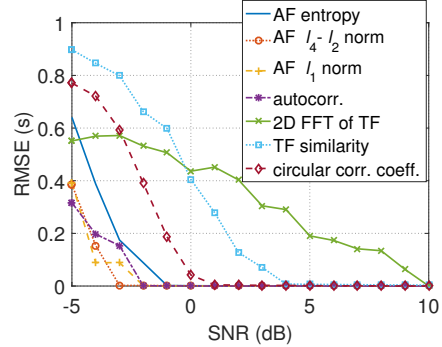

(c)

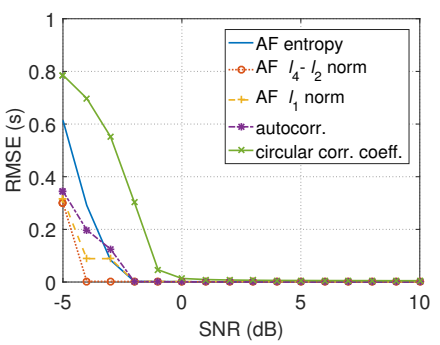

(d)

Fig. 7. Bias and RMSE versus SNR. (a) Bias for the MMLT case; (b) Bias for the MMQT case; (c) RMSE for the MMLT case; (d) RMSE for the MMQT case. 
where $T_{M}$ is the true m-D period, $T_{i}$ is the m-D period estimate in the $i$ th realization, and $I$ is the realization times for each SNR. Both the Bias and the RMSE of the employed approaches decrease as the increase of the SNR. In addition, they are approaching to zero at high SNR.

The performances of these methods for the MMLT case are examined firstly. Among the three AF concentration statistics, the $\mathrm{AF} l_{4}-l_{2}$ norm and $\mathrm{AF} l_{1}$ norm have comparable performance at low SNR (i.e., from $-5 \mathrm{~dB}$ to $-1 \mathrm{~dB}$ ), while the AF entropy performs worse. The performance of the autocorrelation is comparable to the $\mathrm{AF} l_{4}-l_{2}$ norm and $\mathrm{AF}$ $l_{1}$ norm. These four approaches are followed by the circular correlation coefficients. The TF similarity and 2D FFT of TF have the worst performance. Specifically, the 2D FFT performs better at low SNR (from -5 to $-3 \mathrm{~dB}$ for the Bias; from -5 to $0 \mathrm{~dB}$ for the RMSE) while this relation is reversed at high SNR. Remarkably, the Bias and RMSE of the estimate of 2D FFT of TF is larger and approaching to zero more slowly than other approaches. The reason why TF similarity performs poor may be that a small patch of TF (i.e., the partial observations) are used for calculating the similarity. As for 2D FFT of TF, it may be that the overall time support (4s) is short and the frequency resolution is limited $(0.25 \mathrm{~Hz}$ in this case), which is detrimental for the frequency estimator.

Similar with the MMLT case, for the MMQT case, the proposed approach outperforms the circular correlation coefficients as well. It is observed that the performance of the proposed approach for the MMQT case is a little worse than that for MMLT case. Since the difference is so small, it is reasonable to conclude that the proposed approach has a comparable performance for the case with a quadratic translation.

Using both the Bias and the RMSE less than 0.1s (i.e., a relative error less than $5 \%$ ) as the criteria to judge if the estimate is successful. The minimum SNRs required for effective estimates are summarized in Table IV, from which we can see that the AF concentration statistics are more robust to noise than other approaches in this example.

The running time of these estimators are presented in Fig. 8. The running environment is an Intel i7-4712MQ CPU cored personal computer with Matlab R2016a. 5 simulations are run for each approach and the averaged running time is calculated. The running time of these approaches for this example can be divided into four groups: i). the autocorrelation has the smallest running time; ii). the $2 \mathrm{D}$ FFT of TF and the TF similarity have comparable running times, ranking as the second computational efficient group for signal length larger than 600 points; iii). the third group contains the AF entropy, the $\mathrm{AF} l_{4}-l_{2}$ norm and the $\mathrm{AF} l_{1}$ norm; iv). the fourth group consisting of the CSD, the circular correlation coefficients, and the autocorrelation with compensation, stands for the most computational expensive approaches.

\section{B. A Walking Human}

The human walk can be modelled as a periodic motion. In a walking cycle, the arms and legs swing periodically and the body's center of gravity moving up and down periodically [12]. These two movements have the same period. This property

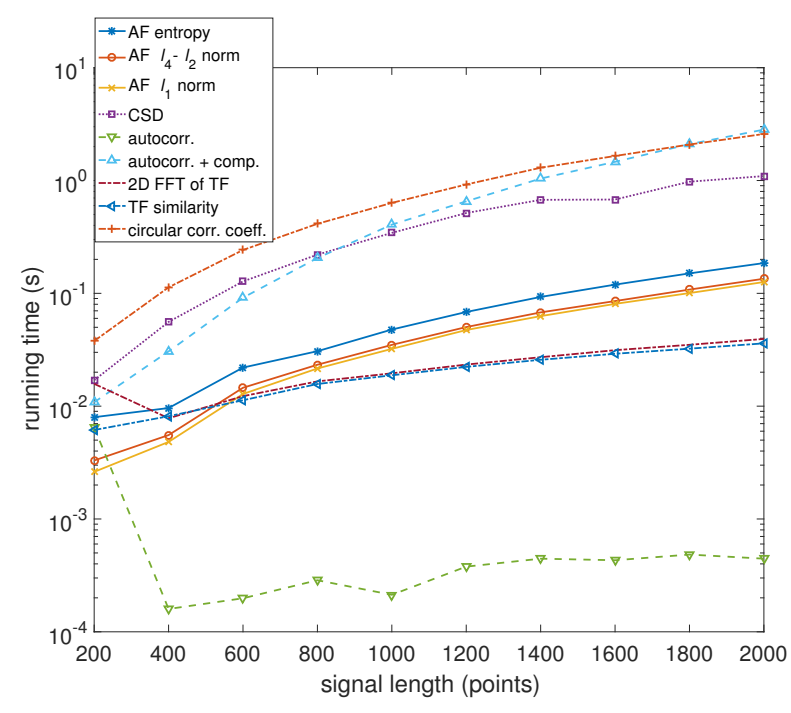

Fig. 8. Running time of the estimation methods.

makes the human walk a special nonrigid micro-motion: most of the m-D period estimation methods can be directly applied to the radar echo of a walking human without a signal decomposition step.

In this part, a m-D signal of a walking human is used to demonstrate the effectiveness of the proposed method. Details about the signal model and its implementation can be found in [12]. The period of gait is $T_{M}=0.5048 \mathrm{~s}$ corresponding to a walking velocity of $0.8101 \mathrm{~m} / \mathrm{s}$. The sampling frequency is $2029 \mathrm{~Hz}$. The signal contains 4 periods. The SNR of the signal is $10 \mathrm{~dB}$. Since the bulk motion of the human can be viewed as constant-velocity motion, the human walk belongs to the MMLT case.

Fig. 9 shows the results of the walking human. The m-D period estimates of these methods are shown in Table V. It can be observed that except the autocorrelation exhibits a weak peak around the m-D period, which is not able to provide an $\mathrm{m}-\mathrm{D}$ estimate, all the methods show peaks around the m-D period and provide high-accurate $\mathrm{m}-\mathrm{D}$ period estimates ${ }^{3}$.

\section{SAR Experiment with a Rotating Target}

Detection and parameter estimation of ground targets based on airborne SAR are of great interest. In this subsection, a rotating target placed on an airport measured by the airborne SAR is used to validate the effectiveness of the aforementioned approaches. The radar works in the sliding spotlight imaging mode. Table VI shows the specifications of the radar system. 10000 pulses are selected for processing and the coherent processing interval (CPI) is $4.9979 \mathrm{~s}$.

Fig. 10 shows the rotating target and SAR image of the interesting area. The rotating target is composed of a rotating bracket, a rotating rod, and two groups of corner reflectors. Each group of corner reflectors contains 4 corner reflectors,

\footnotetext{
${ }^{3}$ In real scenarios, the human m-D signal may be mixed with background inteferences, such as walls and other nearby objects. As a result, the periodicity may be weaken. If the SNR of the signal is too low, the proposed method may fail to extract the period.
} 


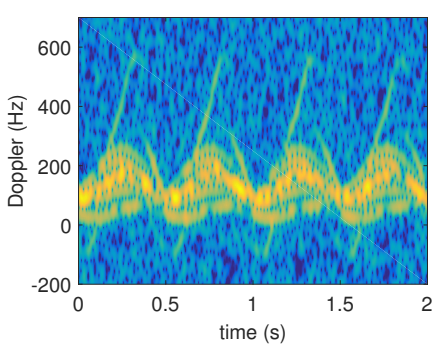

(a)

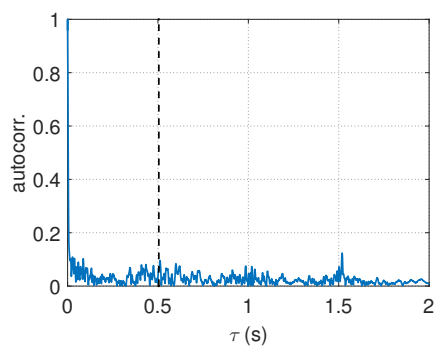

(e)

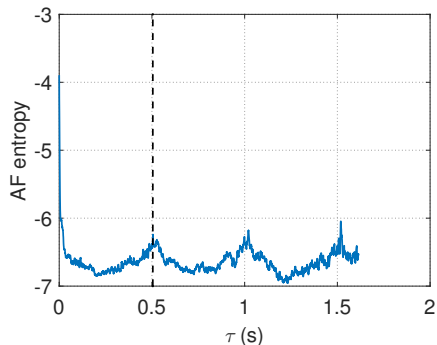

(b)

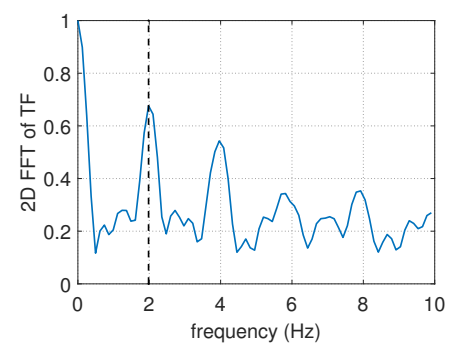

(f)

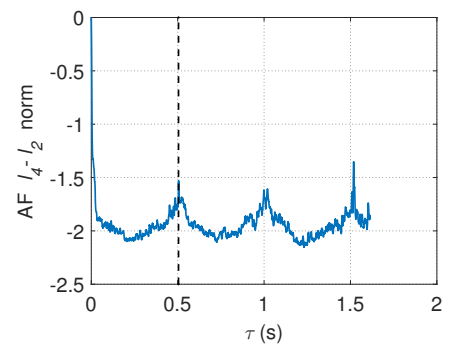

(c)

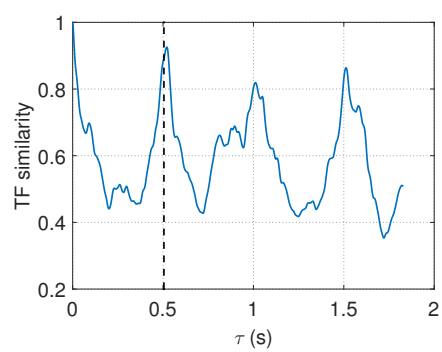

(g)

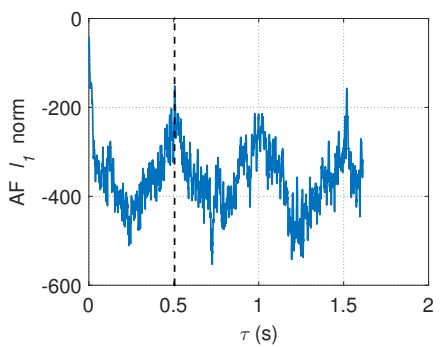

(d)

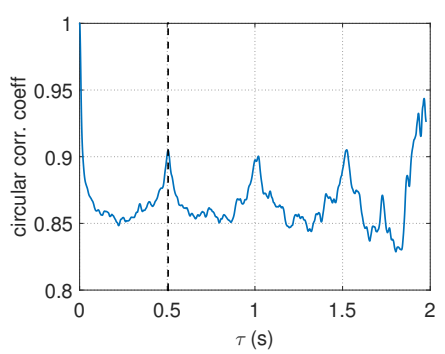

(h)

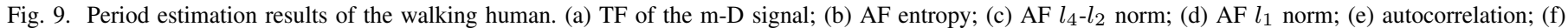
2D FFT of TF; (g) TF similarity; (h) circular correlation coefficients. The black dashed vertical line in the figure has the same meaning with that of Fig. 6.

TABLE V

M-D Period Estimates (s) OF THE WALKing Human

\begin{tabular}{cccccccc}
\hline \hline Human example & $\mathrm{AF}$ entropy & $\mathrm{AF} l_{4} l_{2}$ norm & $\mathrm{AF} l_{1}$ norm & autocorr. & 2D FFT of TF & TF similarity & circular corr. coeff \\
\hline Estimates & 0.5057 & 0.5057 & 0.5052 & na & 0.5048 & 0.5205 & 0.5062 \\
\hline
\end{tabular}

TABLE VI

SPECIFICATIONS OF THE RADAR SYSTEM

\begin{tabular}{lc}
\hline \hline Radar waveform & LFM pulse \\
Center frequency & $9.6 \mathrm{GHz}$ \\
Bandwidth & $1.4 \mathrm{GHz}$ \\
Downlook angle & $66^{\circ}$ \\
Pulse repetition frequency & $2000.64 \mathrm{~Hz}$ \\
Azimuth length of the antenna & $0.546 \mathrm{~m}$ \\
\hline
\end{tabular}

which can reduce the varying of backscatter coefficients induced by different radar incidence angles to some extent. The length of rotating rod is $1 \mathrm{~m}$ which means the rotating radius of the corner reflectors is $0.5 \mathrm{~m}$. The rotating speed is about $25 \mathrm{rpm}$.

Compared with the coning target simulation (which is free of ground clutter), there are two differences in this SAR experiment. Firstly, the m-D signal of the target is mixed with ground clutter which reduces the signal-to-clutter ratio (SCR) of the $\mathrm{m}-\mathrm{D}$ signal. Secondly, the instantaneous Doppler has a linear frequency component due to the motion of the SAR platform which deteriorates the performance of some m-D processing approaches. To alleviate these two effects, we propose a m-D signal extraction method for SAR data which is composed of multistage signal extraction and compensation. The flowchart is shown in Fig. 11 and its details are listed as follows. Step i)iii) are designed to obtain the range-compressed signal of the rotating target. Note that these three steps are not essential and can be replaced by a range compression step. Since the aim of the experiment is mainly to validate the m-D estimation methods, we prefer an easy processing scheme: the target region is selected manually and the bandpass filter in Step vi) is designed to remove the ground clutter. In the future research, an automatic target selection process can be considered.

i). SAR imaging. The imaging algorithm ${ }^{4}$ adopted in this experiment is the chirp scaling algorithm (CSA) [20]. Since the parameters used by SAR imaging is unmatched for rotating targets, the target is defocused and spreads over the entire azimuth bins as shown in Fig. 10(b).

ii). Image cutting. As we are only interested in the analysis of the rotating target, the region from 16902 to $17491 \mathrm{~m}$ in the SAR image is extracted.

iii). Inverse azimuth compression. Transforming back the image of the extracted region to time domain by applying inverse azimuth compression, a range-compressed and azimuthuncompressed signal is obtained. The result is displayed in Fig. 12(a). Range migration ${ }^{5}$ corresponding to the micro-motion of the corner reflectors can be observed. Since the micro-motion of the corner reflectors and the motion of the SAR platform are mixed and there is a range migration correction (RMC) step which is unmatched with the motion of the corner reflectors

\footnotetext{
${ }^{4}$ Range compression and azimuth compression are performed on the received SAR data so that a two-dimensional image of the illuminated scene can be obtained.

${ }^{5}$ The range migration appears if the range variation of the target is larger than the range resolution. For micro-motion target, this effect is referred to as micro-Range effect.
} 


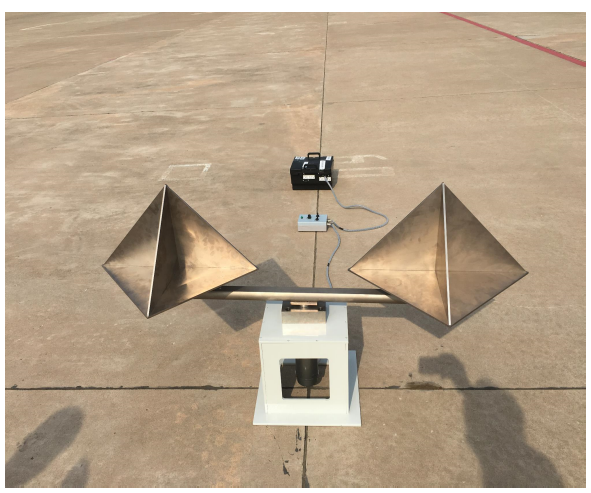

(a)

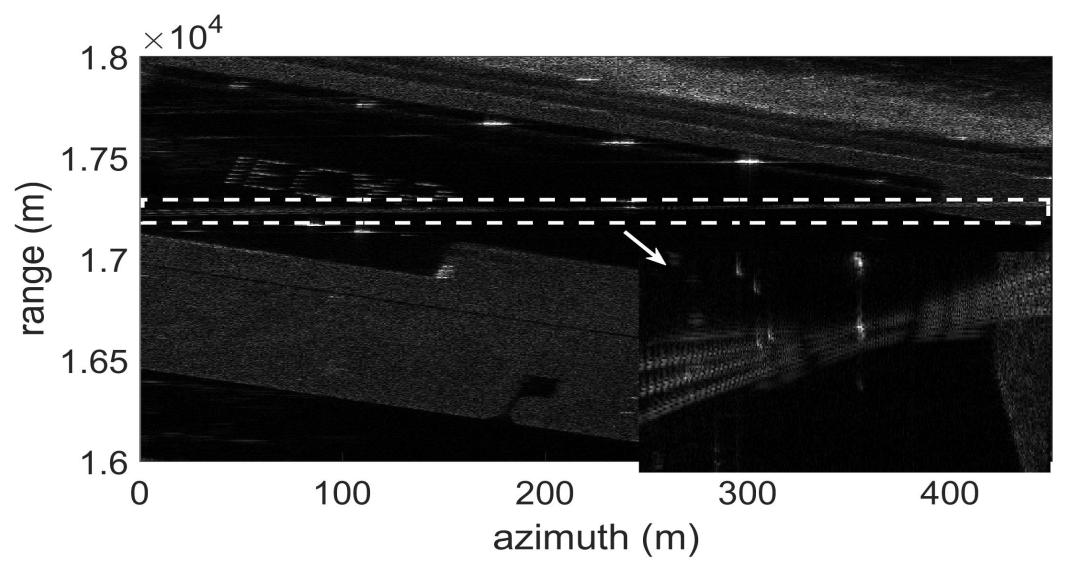

(b)

Fig. 10. The rotating corner reflectors located in the airport. (a) the rotating corner reflectors; (b) SAR image of the interesting area.

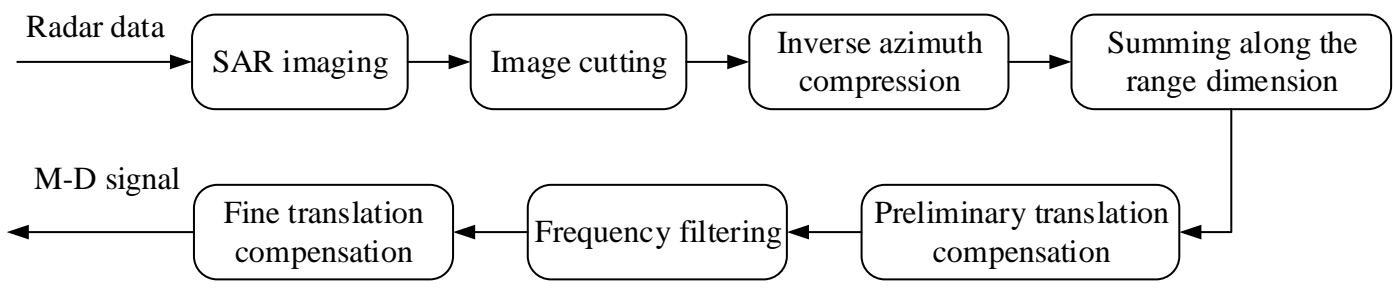

Fig. 11. Flowchart of m-D signal extraction for SAR data.

in the CSA, the range migration of these two corner reflectors is not anymore a standard sinusoidal curve. The signals of the rotating bracket and the rotating rod are weak and cannot be observed in this image.

iv). Summing along the range dimension. As the target spread over multiple range bins, we extract the azimuth signal by simply summing along the range dimension. This step is performed at the price of involving more ground clutter and the range resolution information is not remained. The STFT of the azimuth signal is plotted in Fig. 12(b).

v). Preliminary translation compensation. To compensate the motion of the SAR platform, a preliminary translation compensation is performed on the obtained azimuth signal.

$$
s_{c}(t)=s(t) \exp \left(-j \pi K_{a} t^{2}\right)
$$

where $K_{a}=-2 V_{a}^{2} / \lambda_{c} \bar{R} ; V_{a}$ is the velocity of the SAR platform; $\bar{R}$ is the median range of the extracted region. As there is a difference between $\bar{R}$ and the range of the rotating center, there is still a residual translation after this step.

vi). Frequency filtering. A bandpass filter is applied on $s_{c}(t)$ to further remove the ground clutter. Fig. 12(c) shows the STFT of the preliminary compensated and filtered azimuth signal, which still has a slight linear frequency component.

vii). Fine translation compensation. Finally, the minimumspectrum-entropy translation compensation is carried out for fine translation compensation, yielding a much better result in Fig. 12(d). The slight liner translation component disappears and the m-D of the target centers at zero. By now, the mD signal of the target is extracted successfully. We also plot the RCS of the target in Fig. 12(e). It is clear that since the
LOS of SAR has a large scope and the influence of the strong clutter, the RCS do not show any periodicity. This is quite different from that of most inverse SAR scenarios and violates the assumption of (9). However, in the case of the non-periodic RCS and the strong ground clutter, this complicated scenario provides a good opportunity to test the m-D feature extracting approaches.

M-D period estimation is then performed and the results are reported in Fig. 13. Comparing with the previous example, it is clear that the non-periodic RCS and strong ground clutter lead to the performance degradation for all the approaches. The autocorrelation and TF similarity fail to capture the periodicity of the m-D signal and cannot provide estimates. Other approaches shows peaks around $1.15 \mathrm{~s}$, which is about half of the true rotating period. The reason why the half-period ambiguity appears is that the rotating target has a perfect symmetric structure and this symmetry is reflected in the TF image and the AF image. Without combining other techniques, it is impossible to resolve this ambiguity for approaches merely exploiting the TF and AF periodicity. Even though the AF and AF concentration statistics show much weaker periodicity, these three AF concentration statistics are still effective to provide the period estimates. The 2D FFT of TF also experiences a performance loss. The peak at the location of m-D frequency is quite small and it looks like the sidelobe of the zero frequency component. The circular correlation coefficients has a better quality in this situation. The correlation coefficients at lags near the true m-D period is larger than 0.85 .

At last, the inverse Radon transform (IRT) [21] is performed on the TF image to resolve the period ambiguity. Denote the 


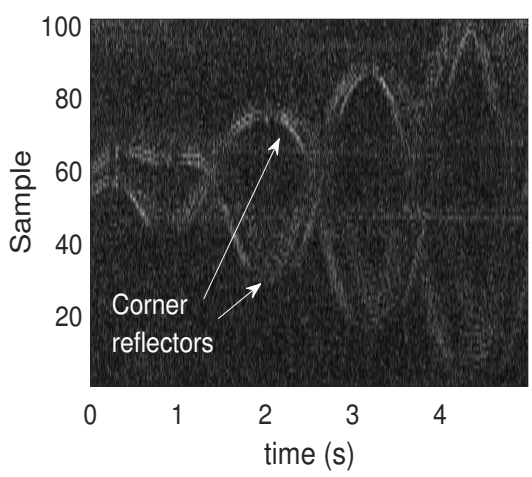

(a)

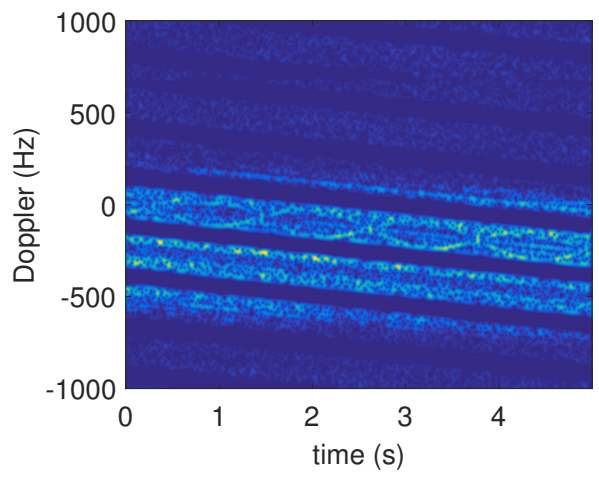

(b)

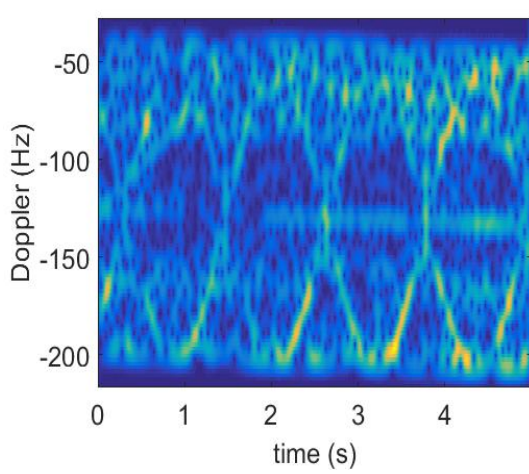

(c)

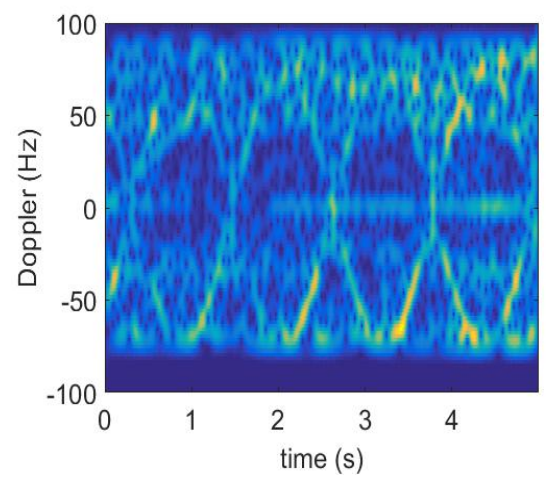

(d)

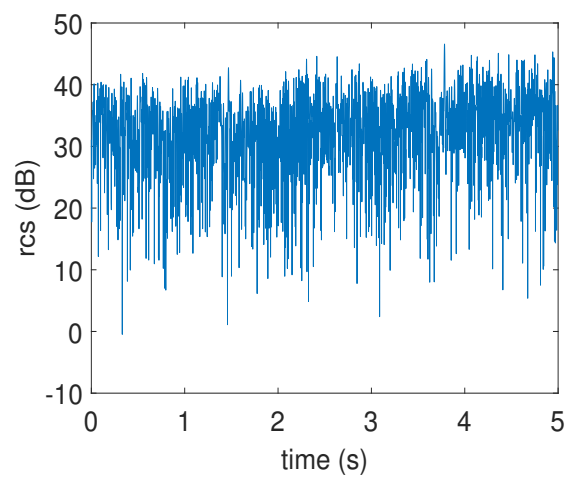

(e)

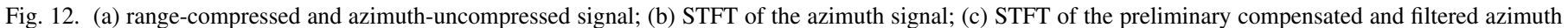
signal; (d) STFT of the m-D signal; (e) the uncalibrated RCS of the target.

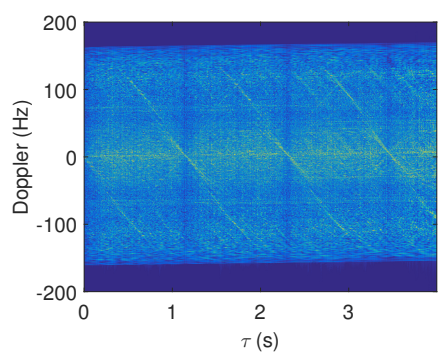

(a)

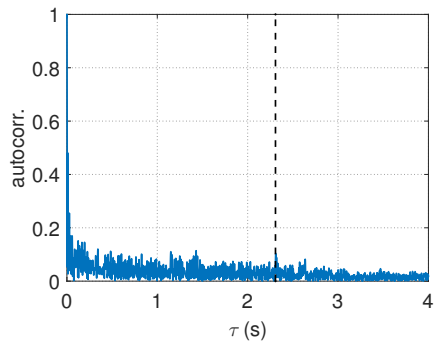

(e)

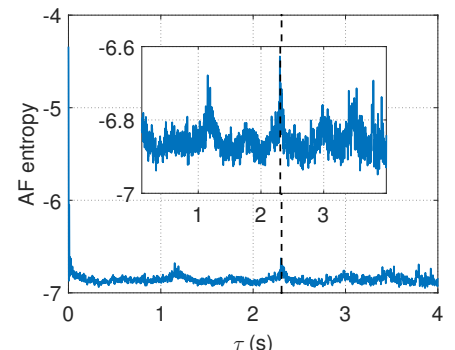

(b)

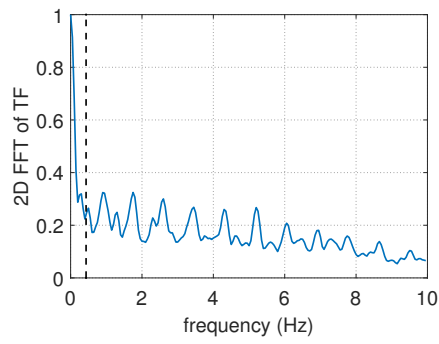

(f)

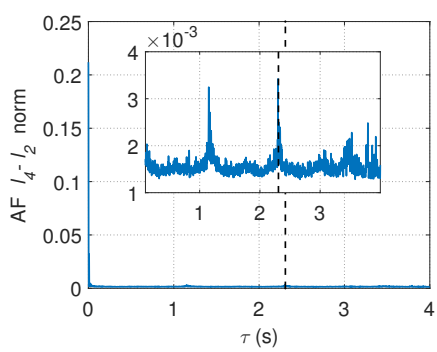

(c)

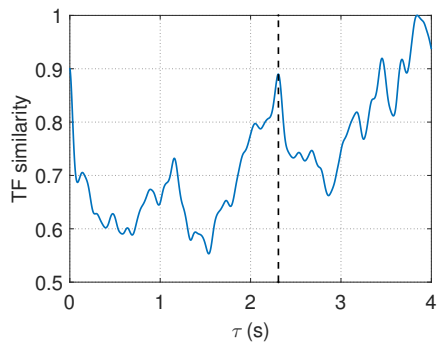

(g)

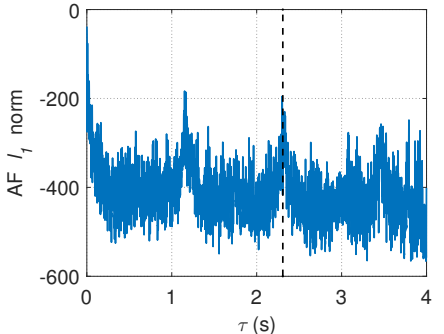

(d)

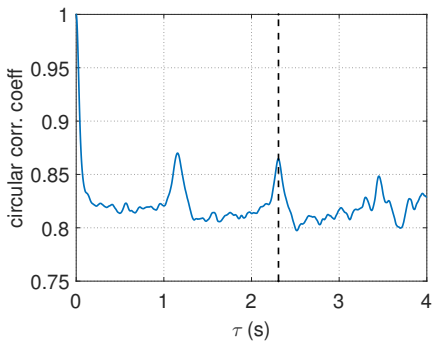

(h)

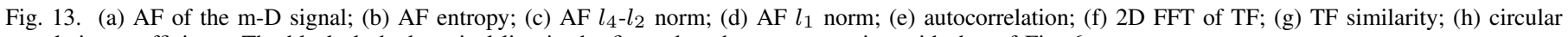
correlation coefficients. The black dashed vertical line in the figure has the same meaning with that of Fig. 6.

initial m-D period estimate of an estimation method as $\hat{T}_{M}^{0}$, the possible m-D period values are $i \hat{T}_{M}^{0}, i=1,2, \cdots$. The IRT with these possible period values are performed respectively.
Since the IRT with true period can detect the sinusoidal curve, it is supposed that the inverse Radon image with true period will have the best focusing effect (which can be measured by 
TABLE VII

M-D Period Estimates (s) OF THE Rotating TARget

\begin{tabular}{cccccccc}
\hline \hline SAR experiment & AF entropy & $\mathrm{AF} l_{4}-l_{2}$ norm & $\mathrm{AF} l_{1}$ norm & autocorr. & 2D FFT of TF & TF similarity & circular corr. coeff \\
\hline Estimates & 2.3023 & 2.3013 & 2.3033 & na & 2.2215 & na & 2.3033 \\
\hline
\end{tabular}

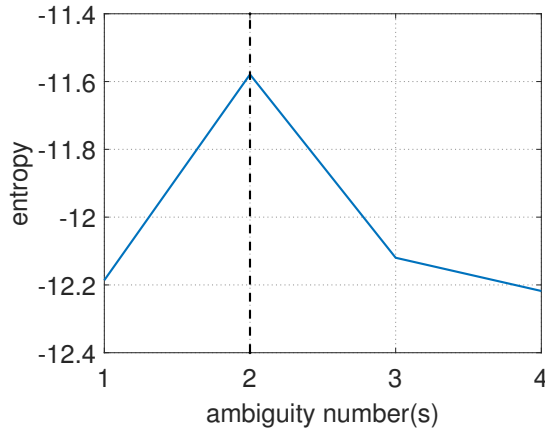

Fig. 14. The entropy of the inverse Radon image. The true ambiguity number is indicated by the black dash vertical line.

the entropy). The true ambiguity number can be estimated by

$$
\hat{i}_{T}=\underset{i}{\operatorname{argmax}} \varepsilon\left[f_{I R}\left(\left|\rho_{s}(t, f)\right|, i \hat{T}_{M}^{0}\right)\right], i=1,2, \cdots
$$

where $f_{I R}\left(\left|\rho_{s}(t, f)\right|, i \hat{T}_{M}^{0}\right)$ denotes the inverse Radon image on $\left|\rho_{s}(t, f)\right|$ with period $i \hat{T}_{M}^{0}$, and $\varepsilon[\cdot]$ is the entropy function.

Taking the initial period estimates of the AF entropy $1.15115 \mathrm{~s}$ as an example, the entropy of the inverse Radon image with respect to the ambiguity number is plotted in Fig. 14. The estimated ambiguity number is 2 and the estimated $\mathrm{m}-\mathrm{D}$ period is $2.3023 \mathrm{~s}$. Fig. 15 shows the inverse Radon image with ambiguity number of 2 and 1 . On one hand, it can be observed from Fig. 15(a) that: when the inverse Radon image is performed with the true m-D period, it has the best focusing. i.e., three scatterers, two represent the two groups of corner reflectors and one represents the rotating bracket, are focused in the inverse Radon image. On the other hand, the image only obtains a focused scatterer in the center and the rest two scatterers are defocused when inverse Radon image is performed with the half period. With the true m$\mathrm{D}$ period, the estimated radii of these scatterers are $0.4643 \mathrm{~m}$, $0.4671 \mathrm{~m}$ and $0 \mathrm{~m}$, respectively. These estimates are close to the true values. All the estimates after resolving the ambiguity number are shown in Table VII. In this context, the IRT has two functions, namely resolving the ambiguity of estimated period and acting as a narrow-band-imaging approach (the two dimensional image is obtained and the sacrificed range resolution is restored).

The major computational cost of this additional processing includes the cost of the TF and the IRT. The IRT is implemented by the filtered backprojection with a computational complexity of $O\left(N N_{f} \log _{2} N_{f}\right)+O\left(N N_{f}^{2}\right)$ [22]. Combined with the computational complexity of the TF which is $O\left(N N_{f} \log _{2} N_{f}\right)$ and use the approximation $\log _{2} N_{f} \ll N_{f}$, the total computational complexity of this additional processing approximates to $O\left(N N_{f}^{2}\right)$. The running time of this additional

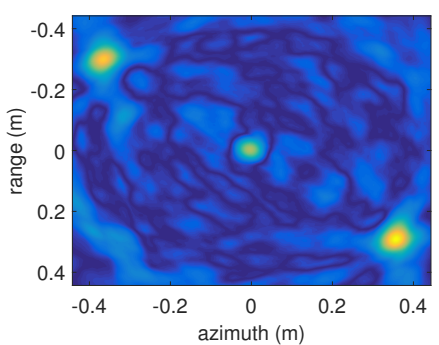

(a)

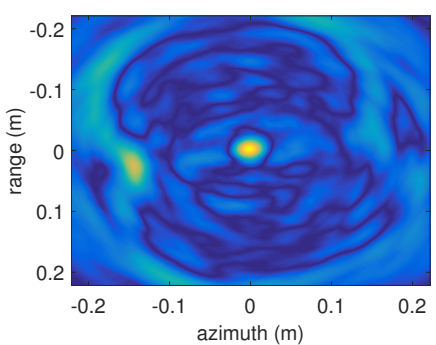

(b)
Fig. 15. Inverse Radon image with different ambiguity number. (a) The ambiguity number is 2 ; (b) The ambiguity number is 1 .

processing (a TF and 4 IRT) in the same computer is $10.72 \mathrm{~s}$ for $N=2500, N_{f}=1001$. The computational cost of the IRT is high. To make it more suitable for real-time processing, it is suggested that the parallel computation or dedicated signal processing hardwares (such as DSP) should be employed.

\section{Discussion}

Three examples, a coning target with two translation cases, a walking human and a rotating target in a SAR experiment, are used to quantify the performance of the AF concentration statistics and make a comparison with other approaches.

Note that in the SAR experiment, all m-D period estimation methods suffer from a loss due to the non-periodic RCS and strong ground clutter. Among these methods, the AF concentration statistics and the circular correlation coefficients are the most robust methods. Moreover, compared with the circular correlation coefficients, the peaks of the AF concentration statistics is less significant in this scenario. Some improvement should be introduced to enhance the periodicity of AF concentration statistics in the future.

From the results, the following conclusions are made. (i). The AF concentration statistics and circular correlation coefficients are (or approximately) invariant to translation with polynomial terms not larger than second order. (ii). By integrating translation compensation, approaches that subjected to the influence of translations (e.g., autocorrelation), can also process the m-D signals with translations. (iii). It is clear that, in addition to time domain and TF domain, m-D period estimation approaches can be applicable in the AF domain. (iv). Concentration statistics in the TF domain is not suitable for capturing the periodicity of m-D signal. (v). The period ambiguity for rotating targets with symmetric structure can be resolved by integrating the IRT. (vi). The proposed method can be directly applied to the radar signal of a walking human to estimate the m-D period. (vii). Among all translation-compensation-free m-D period methods, the AF concentration statistics have the 
best performance, namely the least computational cost and the minimum effective SNR.

\section{CONCLUSION}

This work focuses on m-D period estimation of radar targets with uniform and single-period micro-motion. Targets of interests include ballistic warhead, helicopter, wind turbines, rotating antenna, human, etc.

Though ambiguity function (AF) has been used as a classic tool in the field of radar signal processing (e.g., waveform design and parameter estimation of polynomial-phase signals), it is proposed for the m-D signal study for the first time. It has been widely believed that, in order to have a better performance for processing the multicomponent nonstationary m-D signal, TFRs without cross-term interference should be employed. Our work presents a different way for the m-D period estimation and proves that the AF is capable of doing this as well although it suffers from the cross-term interference.

This work also enlarges the class of the m-D period estimation methods that are free of translation compensation. The AF of m-D signal is analysed elaborately. Three properties of the AF are uncoverd: (i). In the presence of translation, the AF of the m-D signal is circular-shifted and the periodicity is replaced by the circular periodicity. (ii). The concentration of the AF is periodic. (iii). The proposed AF concentration statistics is invariant to translation with polynomial terms not larger than second order. Thus, the proposed method is effective for the $\mathrm{m}-\mathrm{D}$ period estimation without combining a translation compensation step.

Three examples, a coning target with two translation cases, a walking human and a rotating target in a SAR experiment, are used to quantify the performance of the the proposed approach. Experimental results shows that AF concentration statistics has the best performance among all the translationcompensation-free m-D period estimation methods, namely the least computational cost and the minimum required SNR. In the case of a walking human, the proposed method can be directly applied to estimate the m-D period. As for the case of rotating targets with symmetric structure, by integrating the inverse Radon transform (IRT), the period ambiguity can be resolved without the prior structural information (e.g., the number and the length of blades).

As shown in this work, the AF is an effective tool to describe the m-D signal. Moreover, AFs of different targets (e.g., cone shaped target, and rotating corner reflectors) are quite different, which means that the feature of the AF has the potential to serve as another m-D feature. Research on how to use the AF feature to assist target classification would be carried out in the future.

\section{APPENDIX A}

\section{COMPUTATIONAL COMPLEXITY ANALYSIS}

In the analysis of the computational complexity, the division and the logarithm operation are regarded as multiples of multiplications. Other operations which are made up of additions (including the addition) operation are neglected as the addition is implemented much faster than the multiplication.
The IAF of a signal : The IAF of a fixed lag $m$ requires $N-m$ multiplications and the total operations the single-sided IAF $(0 \leq m \leq N-1, N$ lags $)$ required is $\sum_{m=0}^{N-1} N-m=$ $N(N+1) / 2$ multiplications. Similarly, the operations that the two-sided IAF $(-(N-1) \leq m \leq N-1,2 N-1$ lags $)$ required is $N^{2}$. The computational complexity of the IAF is $O\left(N^{2}\right)$.

The FFT of a signal : Assuming the points of FFT is $N_{f}$, it requires $\left(N_{f} \log _{2} N_{f}\right) / 2$ multiplications. The computational complexity of the FFT is $O\left(N_{f} \log _{2} N_{f}\right)$.

The 1D FFT of a matix : Assuming that the size of the matrix is $M \times N$ and we perform 1D FFT along the column with $N_{f}$ points, the operations required is $\left(N N_{f} \log _{2} N_{f}\right) / 2$ multiplications. The computational complexity is $O\left(N N_{f} \log _{2} N_{f}\right)$.

The 2D FFT of a matrix : 2D FFT is achieved by two separated 1D FFT on both the column and the row. Assuming that the matrix is zero-padded to size of $N_{f, 1} \times N_{f, 2}$, the computation required is $N_{f, 1} N_{f, 2}\left(\log _{2} N_{f, 1}+\log _{2} N_{f, 2}\right) / 2$ multiplications and its computational complexity is $O\left(N_{f, 1} N_{f, 2}\left(\log _{2} N_{f, 1}+\log _{2} N_{f, 2}\right)\right)$.

The AF of a signal : The computation of the AF consists of the computation of the IAF and an 1D FFT of the IAF along the time dimension. Thus, the operations that the single-sided AF required are $N\left(N+1+N_{f} \log _{2} N_{f}\right) / 2$ multiplications, and the operations that the two-sided AF required are $1 / 2$. $(2 N-1) N_{f} \log _{2} N_{f}+N^{2}$ multiplications. Assuming that $N_{f}$ is with the same order of $N$, the computational complexity of the AF is $O\left(N^{2} \log _{2} N\right)$.

The STFT of a signal : The computation of the STFT consists of two stages, i.e., multiplied the signal by the window function and an Fourier transform. The total operations required are $N\left(l_{w}+1 / 2 N_{f} \log _{2} N_{f}\right)$ multiplications, where $l_{w}$ is the window length. The spectrogram which is the square of the STFT requires an additional $N N_{f}$ additions. Since $l_{w} \leq N_{f}$, the computational complexity of STFT and the spectrogram are $O\left(N N_{f} \log _{2} N_{f}\right)$.

The entropy of a signal : The entropy of a signal with length $N_{f}$ involves $2 N_{f}$ multiplications, $2 N_{f}-2$ additions, $N_{f}$ divisions and $N_{f}$ logarithm operations. The computational complexity is $O\left(N_{f}\right)$.

The $l_{4}-l_{2}$ norm of a signal: The $l_{4}$ to $l_{2}$ norm of a signal with length involves $2 N_{f}$ multiplications, $2 N_{f}-2$ additions and $N_{f}$ divisions. The computational complexity is $O\left(N_{f}\right)$.

The normalized $l_{1}$ norm of a signal : The normalized $l_{1}$ norm of a signal involves $N_{f}$ divisions and $N_{f}-1$ additions. The computational complexity is $O\left(N_{f}\right)$.

\section{APPENDIX B}

\section{SPECTRum CONCENTRATION STATISTICS FOR Signals WITH DIFFERENT TIME SUPPORT}

Assuming that there are two signals $y(t)$ and $y^{\prime}(t)$, and their spectra $Y(f)$ and $Y^{\prime}(f)$ satisfy the following relationship

$$
\left|Y^{\prime}(f)\right|=T_{s}\left|Y\left(T_{s} f\right)\right|
$$

where $T_{s}$ is the time-support scale. The $l_{p}$ norm of $Y(f)$ is

$$
|Y(f)|_{p}=\int_{-\infty}^{\infty}|Y(f)|^{p} d f
$$


Thus,

$$
\left|Y^{\prime}(f)\right|_{p}=\int_{-\infty}^{\infty}\left|Y^{\prime}(f)\right|^{p} d f=\int_{-\infty}^{\infty} T_{s}^{p}\left|Y\left(T_{s} f\right)\right|^{p} d f=T_{s}^{p-1}|Y(f)|_{p}
$$

The primitive entropy of $Y(f)$ is

$$
\begin{aligned}
\varepsilon_{Y^{\prime}} & =\int_{-\infty}^{\infty} \frac{\left|Y^{\prime}(f)\right|^{2}}{E_{Y^{\prime}}} \ln \frac{\left|Y^{\prime}(f)\right|^{2}}{E_{Y^{\prime}}} d f \\
& =\int_{-\infty}^{\infty} \frac{T_{s}\left|Y\left(T_{s} f\right)\right|^{2}}{E_{Y}} \ln \frac{T_{s}\left|Y\left(T_{s} f\right)\right|^{2}}{E_{Y}} d f \\
& =\varepsilon_{Y}+\ln T_{s}
\end{aligned}
$$

where $E_{Y^{\prime}}=\left|Y^{\prime}(f)\right|_{2}=T_{s}|Y(f)|_{2}=T_{s} E_{Y}$

In order to compensate the effect of varying time support, the redefined entropy is expressed as

$$
\varepsilon_{Y}=\int_{-\infty}^{\infty} \frac{|Y(f)|^{2}}{E_{Y}} \ln \frac{|Y(f)|^{2}}{E_{Y}} d f-\ln T_{y}
$$

where $T_{y}$ is the time support of $y(t)\left(T_{y}\right.$ can be also viewed as the scale to a unit time-support signal).

The ratio of $l_{4}$ norm to the square of $l_{2}$ norm [19] is another widely adopted concentration statistics, defined as

$$
|Y(f)|_{4,2}=\frac{1}{T_{y}}|Y(f)|_{4} /(|Y(f)|)^{2}
$$

Particularly, the normalized $l_{1}$ norm [23] which measures the sparsity can also be used as a concentration statistics,

$$
|Y(f)|_{1, \infty}=-T_{y} \int_{-\infty}^{\infty}|Y(f)| / \max |Y(f)| d f
$$

\section{ACKNOWLEDGMENT}

The authors thank Department of Space Microwave Remote Sensing System, Institute of Electronics, Chinese Academy of Sciences for providing the opportunity to cooperate on the SAR experiment.

This work is supported by the National Natural Science Foundation of China under Grant 61871384 and 61401486, the Natural Science Foundation of Hunan Province, China, under Grant 2017JJ3376, and the Shanghai Aerospace Science and Technology Fund under Grant SAST2017-048.

\section{REFERENCES}

[1] V. C. Chen, F. Li, S. S. Ho, and H. Wechsler, "Micro-Doppler effect in radar: phenomenon, model, and simulation study," IEEE Transactions on Aerospace and Electronic Systems, vol. 42, no. 1, pp. 2-21, Jan 2006.

[2] P. Molchanov, K. Egiazarian, J. Astola, A. Totsky, S. Leshchenko, and M. P. Jarabo-Amores, "Classification of aircraft using micro-Doppler bicoherence-based features," IEEE Transactions on Aerospace and Electronic Systems, vol. 50, no. 2, pp. 1455-1467, April 2014.

[3] P. Lei, J. Wang, P. Guo, and D. Cai, "Automatic classification of radar targets with micro-motions using entropy segmentation and timefrequency features," AEU - International Journal of Electronics and Communications, vol. 65, no. 10, pp. 806 - 813, 2011.

[4] J. Li and H. Ling, "Application of adaptive chirplet representation for ISAR feature extraction from targets with rotating parts," IEE Proceedings - Radar, Sonar and Navigation, vol. 150, no. 4, pp. 284-291, Aug 2003.

[5] T. Thayaparan, S. Abrol, and E. Riseborough, "Micro-Doppler feature extraction of experimental helicopter data using wavelet and timefrequency analysis," in International Conference on Radar Systems, 2004.
[6] C. Cai, W. Liu, J. S. Fu, and L. Lu, "Empirical mode decomposition of micro-Doppler signature," in IEEE International Radar Conference, May 2005, pp. 895-899.

[7] L. Stankovic, T. Thayaparan, M. Dakovic, and V. Popovic-Bugarin, "Micro-Doppler removal in the radar imaging analysis," IEEE Transactions on Aerospace and Electronic Systems, vol. 49, no. 2, pp. 1234-1250, April 2013.

[8] P. Molchanov, J. Astola, K. Egiazarian, and A. Totsky, "On microDoppler period estimation," in 2013 19th International Conference on Control Systems and Computer Science, May 2013, pp. 325-330.

[9] L. Liu, D. McLernon, M. Ghogho, W. Hu, and J. Huang, "Ballistic missile detection via micro-Doppler frequency estimation from radar return," Digital Signal Processing, vol. 22, no. 1, pp. 87 - 95, 2012.

[10] M. Dakovi, M. Brajovi, T. Thayaparan, and L. Stankovi, "An algorithm for micro-Doppler period estimation," in 2012 20th Telecommunications Forum, Nov 2012, pp. 851-854.

[11] K. Li, Y. Liu, K. Huo, W. Jiang, X. Li, and Z. Zhuang, "Estimation of micro-motion parameters based on cyclostationary analysis," IET Signal Processing, vol. 4, no. 3, pp. 218-223, June 2010.

[12] V. C. Chen, The micro-doppler effect in radar. 685 Canton St. Norwood, MA 02062 USA: Artech House, 2011.

[13] X. Li, B. Deng, Y. Qin, H. Wang, and Y. Li, "The influence of target micromotion on SAR and GMTI," IEEE Transactions on Geoscience and Remote Sensing, vol. 49, no. 7, pp. 2738-2751, July 2011.

[14] W. Yu and J. Wang, "Phase adjustment for extraction of micro-motion information of ballistic targets," in 5th International Congress on Image and Signal Processing, Oct 2012, pp. 1837-1840.

[15] W. Zhang, K. Li, and W. Jiang, "Parameter estimation of radar targets with macro-motion and micro-motion based on circular correlation coefficients," IEEE Signal Processing Letters, vol. 22, no. 5, pp. 633637, May 2015.

[16] W. Zhang, K. Li, and W. Jiang, "Micro-motion frequency estimation of radar targets with complicated translations," AEU - International Journal of Electronics and Communications, vol. 69, no. 6, pp. 903 - 914, 2015.

[17] W. Zhang, Y. Fu, L. Nie, G. Zhao, W. Yang, and J. Yang, "Parameter estimation of micro-motion targets for high-range-resolution radar using high-order difference sequence," IET Signal Processing, vol. 12, no. 1, pp. 1-11, 2018.

[18] I. Djurovic, M. Simeunovic, S. Djukanovic, and P. Wang, "A hybrid CPF-HAF estimation of polynomial-phase signals: Detailed statistical analysis," IEEE Transactions on Signal Processing, vol. 60, no. 10, pp. 5010-5023, Oct 2012.

[19] L. Stankovi, "A measure of some timefrequency distributions concentration," Signal Processing, vol. 81, no. 3, pp. 621-631, 2001.

[20] I. G. Cumming and F. H. Wong, Digital Signal Processing of Synthetic Aperture Radar Data: Algorithms and Implementation. 685 Canton St. Norwood, MA 02062 USA: Artech House, 2005.

[21] X. Bai, F. Zhou, M. Xing, and Z. Bao, "High resolution ISAR imaging of targets with rotating parts," IEEE Transactions on Aerospace and Electronic Systems, vol. 47, no. 4, pp. 2530-2543, October 2011.

[22] P. Toft, "The radon transform: Theory and implementation," Ph.D. dissertation, Technical University of Denmark, Denmark, 1996.

[23] R. Gribonval and M. Nielsen, "Sparse representations in unions of bases," IEEE Transactions on Information Theory, vol. 49, no. 12, pp. 33203325, Dec 2003.

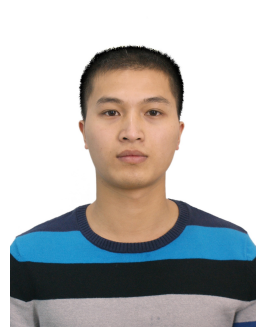

Wenpeng Zhang received the B.S. and Ph.D. degrees in information and communication engineering from the National University of Defense Technology (NUDT), Changsha, China, in 2012 and 2018, respectively. $\mathrm{He}$ is currently a assistant professor with College of Electronic Science, NUDT. His research interests include radar signal processing, timefrequency analysis, and signal representation. 


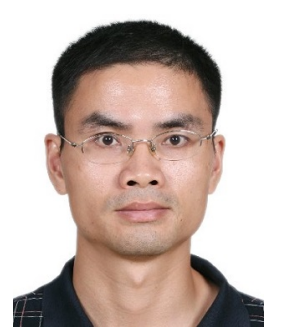

Yaowen Fu received the B.S. and Ph.D. degrees in information and communication engineering from the National University of Defense Technology (NUDT), Changsha, China, in 1997 and 2003, respectively. He is currently a professor with College of Electronic Science, NUDT. His research interests include radar imaging, radar signal processing, target detection, tracking and recognition.

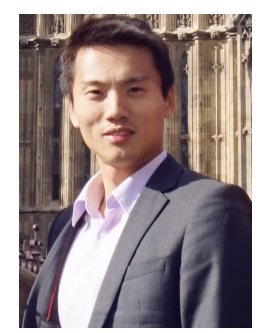

Jiapeng Yin (S'16) received the B.S. degree in information engineering from the National University of Defense Technology, Changsha, China, in 2012. $\mathrm{He}$ is currently working towards the Ph.D. degree at the department of Geoscience and Remote Sensing, Delft University of Technology, the Netherlands. His research interests include radar polarimetry, polarimetric weather radar, radar calibration and radar micro-Doppler. 